\title{
A multigrid AMR algorithm for the study of magnetic reconnection
}

\author{
D. Del Sarto ${ }^{\mathrm{a}, \mathrm{b}}$, E. Deriaz ${ }^{\mathrm{a}, \mathrm{c}}$ \\ ${ }^{a}$ Institut Jean Lamour, CNRS UMR 7198 \\ ${ }^{b}$ Université de Lorraine, Nancy \\ ${ }^{c}$ Centre national de la recherche scientifique
}

\begin{abstract}
We address the study of reconnecting instabilities in magnetized plasmas, a highly multiscale process, using an innovative adaptive scheme based on Adaptive Mesh Refinement (AMR) and Multigrid Algorithms. The reduced model we exploit is very sensitive to numerical errors and demands high order solvers which we develop for this purpose. We validate our approach with two numerical experiments of physical interest.
\end{abstract}

Keywords: Reduced Magnetohydrodynamic, Adaptive Mesh Refinement, Magnetic Reconnection, Multigrid, CFL stability 2000 MSC: 65Z05, 65D05

\section{Introduction}

Through a global change of the magnetic topology, which locally occurs where spatial and/or temporal gradients of macroscopic quantities, such as density current, magnetic field or anisotropic pressure components become important, magnetic reconnection [1] emphasizes the multi-scale character usually displayed by plasma instabilities. The fact that the nonlinear dynamics of reconnection instabilities typically remains confined in a relatively small region of the macroscopic domain makes Adaptive Mesh Refinement (AMR) techniques of integration particularly suited to numerically investigate this problem. To this purpose, they have been already used in many previous studies, both in fluid and kinetic frameworks, among which we mention Refs.[2, 3, 4, 5, 6, 7, 8, 9, 10][11].

Here we present a new AMR algorithm that integrates a set of reduced magnetohydrodynamics (RMHD) equations (see later) by adopting a wavelet- 
based detection of areas of interest for the mesh refinement [12]. The strong points of this algorithm are its high order -three in space while most of the classical AMR schemes are of second order [13, 14, 15]-, and its waveletbased refinement criterion, allowing a stable automatic refinement of the mesh along the areas of interest. We however note that high order AMR Finite Volumes exist which only recently have been made possible by high order Riemann solvers with exact divergence-free condition on unstructured meshes $[16,11]$. The purpose of the algorithm is to allow the study of reconnection at more "realistic" values of the microscopic, non-ideal parameters, which drive reconnection by weighing the gradients of the macroscopic quantities that violate the conservation of the magnetic topology in an ideal MHD plasma. The smallness of such parameters implies indeed an asymptotically small ratio $\delta / a$ between the characteristic linear reconnection layer width, $\delta$, inside of which increasingly smaller spatial scales are nonlinearly generated, and the equilibrium magnetic shear length, $a$. In particular, it always results, both in the classical tearing mode theory and in steady state reconnection, that $\delta / a$ scales as $\sim \varepsilon^{\sigma}$, where $\sigma$ is a positive fractional power of some macroscopic dimensionless parameter $\varepsilon \ll 1$ and usually $1 / 3 \lesssim \sigma \leq 1$ (see e.g. [17]). Realistic values of the latter, for example in a tokamak where $a$ is of the order of the macroscopic length scale $L_{0}$, may well lay in the range $10^{-11} \lesssim \varepsilon \lesssim 10^{-6}$, depending on the regime of reconnection considered (e.g. if purely resistive or purely inertia-driven -cf. Table 1 of Ref.[18]). Therefore, even in a case in which $\delta / a \sim 0.1$ (see the numerical experiment Sec.4.1), a spatial resolution of the inner layer corresponding to $2^{2 \times 12}=4096^{2}$ and of just $2^{2 \times 5}=32^{2}$ points at the macroscopic scales outside of it, means a reduction of a factor 26 in the computational cost with respect to spectral-like codes such as those used for analogous studies of primary reconnection instabilities $[17,19,20,21,22,23,24]$. The fact that the AMR scheme refines only when the singularity appears (and coarsens the grid when a lower resolution results sufficient), makes the adaptivity even more advantageous since almost $70 \%$ of the time the simulation -until the transition to the turbulent nonlinear regime- advances with an accuracy equivalent to a $2^{2 \times 10}=1024^{2}$ points uniform grid while using less than 155818 points. The gain with respect to codes based on non-uniform grids which do not evolve in time (see Refs. $[25,26,27]$ ) becomes then particularly evident in the low-collision regimes relevant to natural and laboratory plasmas, where the smallness of the dissipation length allows a progressive development of short spatial scales. 
The paper is structured as follows. In Sec.1.1 we recall some hot, open problems in magnetic reconnection, affordable in the framework of a RMHD description, for which AMR codes result particularly suited. In Sec.2 the RMHD equations are introduced (Sec.2.1) and the AMR algorithm is described (Sec.2.2-2.4), together with the schemes applied for the integration. An example of fourth order multi-grid Poisson-solver adapted to a nonuniform mesh is detailed in Sec.2.5. In Sec.2.6 the CFL conditions for the integration of the RMHD equations are obtained and discussed in different reconnection regimes. In Sec.3 the physical model behind the equations of Sec.2.1 is recalled, as well as the notion of reconnection instability and the classifications of its different wave-length regimes in terms of the so-called $\Delta^{\prime}$ stability parameter (Sec.3.1). Sec.3.2 focusses on the dissipation-less limit of the model equations, whose Hamiltonian character and implications, both analytical and numerical, are discussed. Sec.4 is devoted to present a few numerical results performed in regimes of collision-less reconnection, already studied in literature, with the aim of both validating the AMR integration scheme and emphasizing its relevance and usefulness: two examples of reconnection leading to a nonlinear "turbulent" (Sec.4.1) and to a "laminar" regime (Sec.4.2) are provided. A conclusion Section follows (Sec.5), in which comments about the parallelization of the algorithm are also given.

\subsection{Relevance and usefulness of RMHD AMR schemes for some up-to-date reconnection problems}

Most numerical studies on magnetic reconnection with AMR schemes are aimed at performing MHD or PIC 3D massive simulations, whereas just few exceptions, like Ref.[6], focus on the exploitation of self-adapting grid schemes for the integration of RMHD equations. From a numerical point of view, the slab geometry RMHD modelling we are going to focus on, is free from the "burden" of the $\boldsymbol{\nabla} \cdot \boldsymbol{B}=0$ constraint, since the divergence-free condition on the magnetic field results automatically satisfied in this framework where flux and stream functions only are considered (see Sec.2.1). From the physical point of view, the RMHD description does not retain the Hall-MHD effects that are responsible, for example, of the transition to a fast reconnection by excitation of whistler waves [28], but is appropriate to describe reconnection instabilities in plasma confined by a strong guide field (e.g. in tokamaks). More generally, RMHD results very useful for isolating the fundamental physics related to the stability and evolution of large aspect ratio current sheets generated by ideal processes or by primary reconnection insta- 
bilities. This dynamics is relevant to phenomena like tokamak disruptions, solar flares or to the magnetic activity in the magnetosphere. In particular, the missing ingredients to look for, in order to understand and model the fast reconnection rates which are suggested by experimental evidence, have been since long time considered the kinetic processes that affect the evolution of current sheets and/or the occurrence of secondary reconnection instabilities, or, in turbulent environments such as the solar corona, effects related to the stochasticity of the turbulent magnetic field fluctuations [29]. A prominent example of kinetic effects expected to speed up the reconnection process is the generation of anomalous resistivity, but many other mechanisms may intervene at microscopic scales comparable to kinetic ones. These effects are believed to suffice to explain the observed fast reconnection rates, even within fluid descriptions. The recent results of Refs.[18, 30, 31] provide models for when this may occur in a RMHD approximation, thus without the need to rely on the transition to the whistler mediated, Hall-reconnection, because of geometrical features of the reconnecting current sheet, which are related to the magnitude of its aspect ratio. An example of such microscopic effects occurring at kinetic-like scales, and which can be retained in the extended fluid model we consider here, is provided by non dissipative reconnection mechanisms due to a non negligible electron inertia [19, 32], first devised in Refs. $[33,34]$ as a likely alternative to resistive reconnection. Fast reconnection instabilities associated to the development of "plasmoids" (i.e., magnetic islands) on large aspect ratio current sheets have been first numerically evidenced in Ref.[35] by Biskamp, but it is only with the work of Ref.[39] that an early theoretical model for fast reconnection has been provided by Shibata and Tanuma, based on the generation of plasmoids on the current sheets generated by primary reconnecting modes. This possibility has been then supported by several early numerical studies of secondary reconnection processes [38, 21, 40, 41], among which it is due to include also the preceding, pioneering works of Refs.[36, 37] (despite less stress or direct evidence was put in the latter on the reconnection rate enhancement due to secondary reconnecting instabilities). The relationship between fast reconnection rates and the occurrence of plasmoids has been later reinterpreted in the light of the "plasmoid instability" scaling $[42,43]$. This, on the other hand, turns out to be nothing but the scaling of a tearing mode developing on a steady Sweet-Parker current sheet, as a previous derivation by Tajima and Shibata had already evidenced [44]. More recently, however, the existence of a quasisingular tearing eigenmode corresponding to an ideal growth rate $\left(\gamma \tau \sim \varepsilon^{0}\right)$, 
and therefore dubbed "ideal" tearing, has been shown in the quasi-ideal limit $\varepsilon \rightarrow 0$ with $\varepsilon \neq 0$, first in the resistive regime [30] and then in reconnection induced by non-collisional effects [18]. In particular, by relying on a rescaling argument such as that first exploited in Ref.[39], it was pointed out in Ref.[30] how the tearing growth rate becomes an increasing function of the current sheet aspect ratio $L_{c s} / a_{c s}$, once the current sheet length $L_{c s}$ is fixed and assumed as the macroscopic reference scale, much larger than the current sheet width, $a_{c s}$. This has opened a new scenario for the description of the reconnection rate increase up to an Alfvénic, "ideal" time scale, during the nonlinear phase of primary tearing-type modes [18, 45, 46, 31], since current sheets nonlinearly generated by primary reconnecting modes on a magnetic field with shear length $a$ easily achieve aspect ratios $L_{c s} / a_{c s} \sim a / \delta \gg 1$ (see Refs. $[17,19]$ and all the numerical studies about secondary reconnection quoted so far). For example, relying on the essence of this re-scaling argument for the reference lengths, a possible theoretical interpretation has been given [31] of the sawtooth crash in tokamaks, where fast reconnecting current sheets (yet not necessarily on an ideal time scale) can be generated by primary reconnection instabilities, in turn too slow to account, alone, for the short time scales of the phenomenon. Evidence of such fast, secondary instabilities in the sawtooth cycle had been earlier provided by numerical simulations of $\mathrm{Yu}$ et al. [47] and, less explicitly, in Refs.[36, 37]. On the other hand, the very existence of the "ideal" tearing solution has questioned the possibility to achieve a Sweet-Parker-type steady reconnection regime both in resistive [30], viscous-resistive [48] and inertial regimes [18]. This has provided an explanation to the instability of a Sweet-Parker current sheet over ideal time scales, earlier evidenced by Biskamp for sufficiently small values of the resistivity [35]. At the same time, the unlikeliness of the Sweet-Parker assumption in weakly collisional plasmas invalidates, in turn, the hypothesis at the basis of the plasmoid instability scaling, which in recent years has been regarded as the most promising paradigm to explain by means of secondary instabilities the fast reconnection rates deduced from measurements (for a recent review on secondary reconnecting instabilities observed in simulations and experiments, and on the notion of fast reconnection, see Ref.[31]). Even if the quasi-singular "ideal" tearing solution is formally obtained in the asymptotic limit $\varepsilon \rightarrow 0$ with $\varepsilon \neq 0$, it is of general, practical relevance to physical phenomena: the values of the "ideal" tearing growth rates, which differ in each reconnection regime depending on the nature of the parameter $\varepsilon$ involved, are achieved with excellent approximation for values of $\varepsilon$ encoun- 
tered in nature or in laboratory $[18,30,48]$. In particular, a quantitative analysis, both theoretical and numerical, of the secondary reconnecting instability occurring on a current sheet whose aspect ratio is rapidly increasing has been first provided by Tenerani et al. in Ref.[46] and recently reviewed in Ref.[49]. These works, which have given the first numerical evidence that is unlikely to achieve a Sweet-Parker steady configuration in a dynamically evolving setting, also show how delicate can be to distinguish in numerical simulations the different scalings predicted by asymptotic theories: in this case, for example, only for sufficiently small values of the plasma resistivity (corresponding to Lundquist numbers $\gtrsim 10^{6}$-see, e.g., Fig.4 of Ref.[46]) it is possible to separate scalings of the current sheet aspect ratio which go like powers $1 / 2$ or $1 / 3$ of the resistivity. But even more challenging, from a computational point of view, can be to quantify the growth rates predicted by asymptotic models for secondary reconnecting instabilities [31] which estimate, for example, scalings like $1 / 6$ or $1 / 7$, against the $1 / 3$ scalings of the primary mode's growth rate.

Remarkable numerical efforts are therefore required to perform nonlinear simulations capable of testing the correctness of the scalings predicted by all these theoretical models, since only for sufficiently small values of $\varepsilon$ the numerically measured scalings can be accurately compared with those predicted by the different asymptotic theories. Only a few recent numerical investigations (see e.g. Refs. $[46,49,50]$ ) have been performed with this purpose in a parameter range $\left(\varepsilon \lesssim 10^{-6}\right)$ which just begins to be significant in this sense, and many open questions still remain to be answered. Examples concern the role of flows and of secondary fluid-type instabilities in the disruption of current sheets $[20,46]$; the role of the relative amplitude of the reconnecting magnetic field with respect to the ambient one [31, 51]; not to speak of the specific features of the current sheets dynamics (amplitude, characteristic spatial and time scales of the current density's evolution) and the way these are related to the onset of secondary reconnecting instabilities. The latter point is crucial to test and investigate one of the main implications of the "ideal" tearing model, that is, the possibility to observe turbulent reconnection processes $[52,29]$ due to the occurrence of secondary tearing modes on the current sheets generated by ideal, convective motions - see in particular the discussion of Ref.[30, 53], the extensive analysis of Refs. [46, 49] and the more recent comments in Refs.[18,31]. These phenomena are of great relevance to turbulent media such as plasmas on the solar corona or in the solar wind, as well as in magnetic confinement experiments, an example 
being provided by drift-kinetic turbulent transport in tokamak plasmas. In these large scale turbulent frameworks, the formation of progressively thinner current sheets due to the fluid convection in an almost collisionless regime $(\varepsilon \rightarrow 0)$, usually requires to numerically quantify the global energy conversion processes, which allow to transfer magnetic energy to thermal heating via magnetic reconnection, in terms of statistical models that account for the microscopic stochastic fluctuations of the magnetic field $[29,54]$. These models, however, rely on specific hypotheses and scalings about the average behaviour and aspect ratio of the single current sheet, which are related to, or depend on, the reconnecting models we have discussed so far. Moreover, also the interplay between the nonlinear dynamics of tearing instabilities and a background turbulence is an issue of general importance for both coronal plasmas [53] and tokamaks [55]. In the latter, for example, magnetic islands generated by primary tearing and kink instabilities in the core plasma may become the seed for neoclassical tearing modes excited by tokamak edge turbulence [56].

It is furthermore worth remarking that, if one wants to investigate the nonlinear dynamics of a primary tearing mode in the "ideal" tearing regime, as it has been done in Refs.[45, 46, 49,50], a huge scale separation $L \gg a \gg \delta$ is needed already in the linear stage of the simulation, especially in the asymptotic regime $\varepsilon \ll 1$, since both the equilibrium current sheet inverse aspect ratio $a / L$ (with $L$ in fact being one size of the simulation box) and the ratio $\delta / a$, between the width of the linear reconnecting layer $\delta$ and the equilibrium current sheet $a$, scale like positive powers of $\varepsilon$.

All those provided above are examples of computationally challenging problems that the AMR algorithm we are going to present can help to efficiently address, while aiming to perform numerical studies of reconnection phenomena with microscopic parameters sufficiently small to approach realistic values.

\section{Adaptive mesh refinement numerical scheme}

\subsection{Model equations}

We have applied the AMR technique to the integration of the following set of RMHD equations (see, e.g., Refs.[17, 18] and references therein for a discussion) for quantities depending on $x, y$ (space) and $t$ (time),

$$
\frac{\partial F}{\partial t}+[\varphi, F]=\rho_{s}^{2}[U, \psi]+\eta\left(\nabla^{2} \psi-\nabla^{2} \psi_{e q}\right)
$$




$$
\begin{gathered}
\frac{\partial U}{\partial t}+[\varphi, U]=\left[\psi, \nabla^{2} \psi\right]+\nu\left(\nabla^{2} U-\nabla^{2} U_{e q}\right), \\
F \equiv \psi-d_{e}^{2} \nabla^{2} \psi \quad U \equiv \nabla^{2} \varphi
\end{gathered}
$$

with $\left(\psi_{e q}\right.$ and $\left.U_{e q}\right)$ denoting the equilibrium quantities and the Poisson brackets meaning, with standard notation,

$$
[f, g] \equiv \nabla f \times \nabla g \cdot \boldsymbol{e}_{z}=\partial_{x} f \partial_{y} g-\partial_{y} f \partial_{x} g
$$

The physical meaning of the RMHD set will be recalled next, when some applications of the numerical model are discussed (Sec.3). For the sake of future notation we remind that the Poisson brackets relate to the convective derivatives associated to the fluid velocity $\boldsymbol{u} \equiv \boldsymbol{e}_{z} \times \boldsymbol{\nabla} \varphi$, and to the in-plane magnetic field, $\boldsymbol{B}_{\perp} \equiv \nabla \psi \times \boldsymbol{e}_{z}$, that is

$$
[\varphi, f]=\boldsymbol{u} \cdot \nabla f, \quad[\psi, f]=-\boldsymbol{B}_{\perp} \cdot \nabla f .
$$

The $z$-components of the vorticity and of the current density are therefore

$$
(\nabla \times \boldsymbol{u})_{z}=\nabla^{2} \varphi=U, \quad(\boldsymbol{\nabla} \times \boldsymbol{B})_{z}=-\nabla^{2} \psi=J_{z}
$$

As it is customary, equilibrium quantities are subtracted in the last r.h.s. terms of Eqs.(1-2), in order to prevent dissipation effects to affect them on time scales numerically comparable to those of the linear instabilities we want to study. This problem would be avoided in the asymptotic limit in which both $\eta$ and $\nu$ are sufficiently close to zero and for which the more realistic equations, without such a subtraction, could be restored. Then we note that the finite differences AMR algorithm developed for Eqs.(13) can be easily modified to integrate equations with analogous differential operators, such as those of the electron-magnetohydrodynamic regime $[57$, 58], and to include further microscopic effects such as ion-Finite Larmor Radius (FLR) corrections [24]. Therefore, it can be easily upgraded to include similar equations for further scalar fields (see, e.g., Refs.[59, 60, 55]), possibly dependent from a third space coordinate [61, 62].

We have implemented two main cases of the RMHD equations $(1,2,3)$ with AMR schemes: the inertial case when $\eta=0$ and $d_{e} \neq 0$ and the resistive case when $\eta \neq 0$ and $d_{e}=0$. Both require high-order numerical integration. While in both implementations some care is needed for the elliptic solvers, 
the first case needs a special attention to be payed to the CFL conditions (Sec.2.6), the second one requires an IMEX scheme [64] in order to solve efficiently the convection terms explicitly and the diffusion term implicitly.

In this paper we focus on the inertial case with $\eta=0$ and $d_{e} \neq 0$, whose nonlinear description is the most challenging from a computational point of view (cf. Sec.3.2 and 4).

\section{2. $A M R$ and finite differences}

As the reconnecting instability develops in a very thin layer along the invariant axis, say $y$, its numerical simulation benefits from an adaptive mesh. The grid refines or coarsens itself according to the spatial regularity of the solution, so only a small portion of the domain is finely meshed. The points allocated inside a tree structure at dyadic positions are recursively split in a fractal way as long as the accuracy of the numerical approximation requires it $[65,69]$. The AMR solver we propose is written in $C$ and allows us to test original computer algorithms and numerical methods.

While most of the existing AMR are based on finite volume methods and cell-centered refinement methods, we propose a finite-differences pointcentered refinement technique. It provides an easy access to high order numerical schemes: typically, we are using an upwind third order finite difference for the convections, and fourth order compact finite-differences multigrid solvers for the elliptic equations (of Poisson type). Until the late 2000's $[13,65,14]$ finite-volume cell-centered schemes hardly reached an accuracy beyond the second order. Only recently high order finite-volume schemes based on Riemann solvers for MHD equations have been made available $[16,11,66,67,68]$.

This high order is essential to simulate the RMHD model Eq. (1), (2) and (3) where we have to compute $\nabla \phi$ and $\nabla \psi$ with a high accuracy in the non uniform grid. The other possibilities to maintain the order of the discrete operators are either to consider a uniform grid with periodic boundary conditions or to express all the differential operators with respect to a unique discrete operator. For instance, when solving $\nabla^{2} u=v$, if the discretized operators $\nabla_{\Delta x}^{2}, \nabla \cdot \Delta_{x x}$ and $\nabla_{\Delta x}$ satisfy $\nabla_{\Delta x}^{2}=\nabla \cdot{ }_{\Delta x} \circ \nabla_{\Delta x}$, as in [65], then $\nabla_{\Delta x}\left(\nabla_{\Delta x}^{2}\right)^{-1} v$ is computed at an order equal to the minimum order of $\boldsymbol{\nabla} \cdot{ }_{\Delta x}$ and $\boldsymbol{\nabla}_{\Delta x}$. Whereas, in our case, the order of $\nabla_{\Delta x}\left(\nabla_{\Delta x}^{2}\right)^{-1}$ equals the minimum between the order of $\nabla_{\Delta x}^{2}$ minus one and the order of $\nabla_{\Delta x}$.

We numerically solve both the elliptic equations (3) by using fourth-order multigrid methods adapted to the non uniform mesh. They follow the pattern 


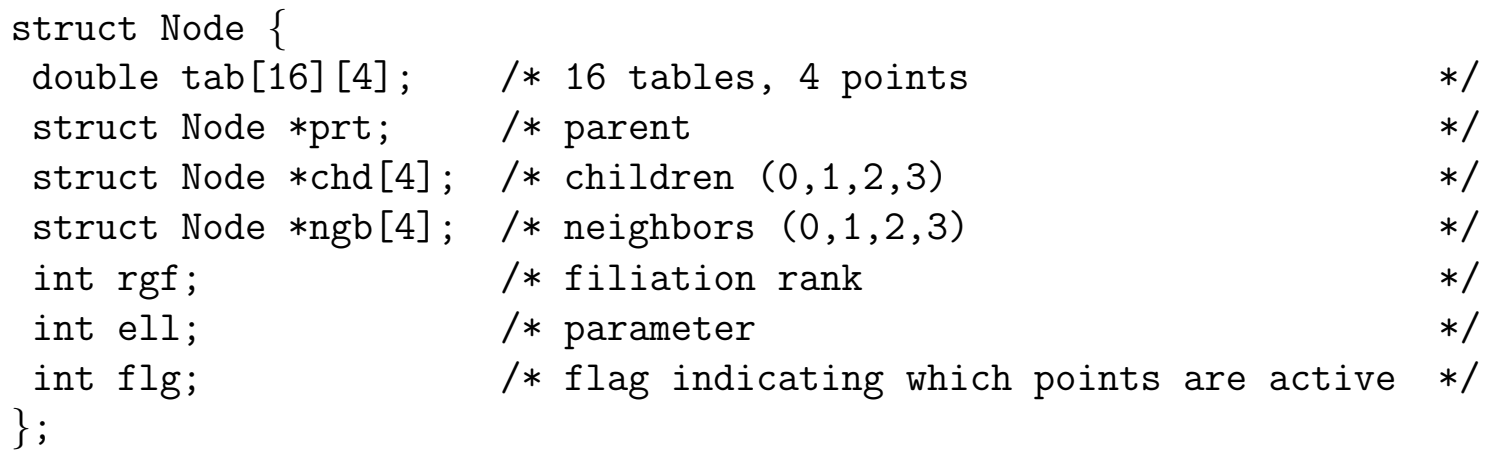

Table 1: The Node: basic element of the tree structure.

detailed in the Sec. 2.5. Aside Eqs.(3) a similar method allows to compute $J_{z}=\nabla^{2} \psi=\nabla^{2}\left(I d-d_{e}^{2} \nabla^{2}\right)^{-1} F$ directly from $F$.

\subsection{Implementation}

The whole implementation is based on a structure named Node presented in Table 1. This structure gathers all the physical values of the simulation and connects to the other nodes in order to form the tree structure. The node at the top of the tree is called the root. Following the parent-to-children links it gives access to the whole tree. In the domain $\left[0, L_{x}\right] \times\left[0, L_{y}\right]$ with periodic boundary conditions, the four points of the root are located at $(0,0)$, $\left(L_{x} / 2,0\right),\left(0, L_{y} / 2\right)$ and $\left(L_{x} / 2, L_{y} / 2\right)$. Each of these points can be refined into four points. For instance the point $(0,0)$ may be refined. In this case, we just allocate a new node which is pointed by chd [0] in the initial root node and which contains the points $(0,0),\left(L_{x} / 4,0\right),\left(0, L_{y} / 4\right)$ and $\left(L_{x} / 4, L_{y} / 4\right)$.

Recursively we generate a grid with points located at positions of the type $\left(2^{-j} \kappa_{1} L_{x}, 2^{-j} \kappa_{2} L_{y}\right)$ for integers $j \geq 1$ and $0 \leq \kappa_{1}, \kappa_{2} \leq 2^{j}-1$.

At each time step, the grid is modified according to the refinement criterion of paragraph 2.4. Then we create a table which lists all the nodes level by level. This table allows to:

1. compute the finite differences level by level starting from the coarsest one. When a point cannot be computed by the usual finite difference scheme because a neighboring point is missing, then it is interpolated by the result obtained at the coarser level,

2. apply multi-grid schemes (Sec.2.5),

3. parallelize these operations with OpenMP. We cut and distribute each loop over each level. 
Regarding the time scheme, we basically apply the fourth order RungeKutta scheme.

\subsection{Mesh refinement criterion}

At the end of each Runge-Kutta time step, the mesh is refined and coarsened according to a simple regularity indicator. For each level $j$ and for each point $2^{-j} \boldsymbol{\kappa}=\left(2^{-j} \kappa_{1}, 2^{-j} \kappa_{2}\right)$ of the level $j$, we compute the residual of a linear interpolation by the coarser level $j-1$ :

$$
d_{j,\left(2 \ell_{1}+1,2 \ell_{2}\right)}=f_{j,\left(2 \ell_{1}+1,2 \ell_{2}\right)}-\frac{f_{j,\left(2 \ell_{1}, 2 \ell_{2}\right)}+f_{j,\left(2 \ell_{1}+2,2 \ell_{2}\right)}}{2},
$$

the case for $d_{j,\left(2 \ell_{1}, 2 \ell_{2}+1\right)}$ is obtained by symmetry, and

$d_{j,\left(2 \ell_{1}+1,2 \ell_{2}+1\right)}=f_{j,\left(2 \ell_{1}+1,2 \ell_{2}+1\right)}-\frac{f_{j,\left(2 \ell_{1}, 2 \ell_{2}\right)}+f_{j,\left(2 \ell_{1}+2,2 \ell_{2}\right)}+f_{j,\left(2 \ell_{1}, 2 \ell_{2}+2\right)}+f_{j,\left(2 \ell_{1}+2,2 \ell_{2}+2\right)}}{4}$.

Remark that $f_{j,\left(2 \ell_{1}, 2 \ell_{2}\right)}=f_{j-1,\left(\ell_{1}, \ell_{2}\right)}$ so there is no residual at this point.

Then we consider a cell-centered estimator around $2^{-j} \boldsymbol{\kappa}=2^{-j}\left(2 \ell_{1}+\right.$ $\left.1,2 \ell_{2}+1\right)$ for all $\ell_{1}$ and $\ell_{2}$ :

$$
w_{j \kappa}^{2}=\sum_{e_{1} \in\{-1,0,1\}} \sum_{e_{2} \in\{-1,0,1\}} d_{j\left(\kappa_{1}+e_{1}, \kappa_{2}+e_{2}\right)}^{2} .
$$

Hence for each $(j, \boldsymbol{\kappa})$ such that $\kappa_{i}=2 \ell_{i}+1$, we know the weight $w_{j \kappa}$ associated to the point $(j, \boldsymbol{\kappa})$. This point is the center of a cell formed by the node that contains it, and its three -right, upper and upper-right- neighboring nodes. For the thresholding the weigh $w_{j \kappa}$ is compared to the quantity $2^{\alpha j} \epsilon$ which depends on the given refinement parameters $\alpha$ and $\epsilon$ and on the refinement level $j$. The parameter $\alpha$ between -2 and $+\infty$ modules the approximation space: if $\alpha=\operatorname{dim} / p-s$ with $\operatorname{dim}$ the space dimension (here $\operatorname{dim}=2$ ) then this thresholding minimizes the $B_{p}^{s}\left(L^{p}\right)$-norm of the error (i.e. the $L^{p}$-norm of its $s$-th derivative) [63]. Hence $\alpha=1$ corresponds to a $L^{2}$-thresholding of the solution, and $\alpha=0$ to a $L^{\infty}$-thresholding.

Then we apply the following refinement and prune algorithm:

- (refine 1) if $w_{j \kappa}>2^{\alpha j} \epsilon$, the children of the nine points around $(j, \boldsymbol{\kappa})$ are activated (created if they do not exist, preserved if they do),

- if $w_{j \kappa}>2^{\alpha j} \epsilon / 2$, then the cell is preserved, the same if it is the parent of a preserved node, 
- (refine 2) the neighboring nodes of the activated nodes up to now are activated on two ranks, this implies the graduation of the tree,

- (prune) at this stage, if a node is not preserved then it is removed,

- in addition, for some of the experiments, we impose a maximum limit to the refinement level: $j \leq j_{\text {lim }}$.

The larger $\alpha$, the closer the grid will be to a uniform grid. The values of $F$ and $U$ of the newly created points are interpolated from the points of the coarser level thanks to a fourth-order interpolation scheme.

\subsection{Multigrid Collatz Poisson solver}

Here we detail a multi-grid Collatz solver applied to the AMR framework. The Collatz method is a fourth order compact finite difference method for solving the Poisson equation $\nabla^{2} u=v$. In the 80's it was applied in the multi-grid context [70, 71]. Then, during the years 2000 and 2010, it was successfully implemented in two and three dimensions for non-uniform grids and for nested grids data structure in the Adaptive Mesh Refinement and Finite Volume frameworks $[72,73,74]$ but not for Fully Threaded Tree structures as presented here.

In dimension two it is given by:

$$
\frac{1}{6 h^{2}}\left[\begin{array}{ccc}
1 & 4 & 1 \\
4 & -20 & 4 \\
1 & 4 & 1
\end{array}\right] u=\frac{1}{12}\left[\begin{array}{lll}
0 & 1 & 0 \\
1 & 8 & 1 \\
0 & 1 & 0
\end{array}\right] v
$$

for a uniform discretization in $x$ and $y$ with space step $h: x_{i_{1}}=i_{1} h, y_{i_{2}}=i_{2} h$. The above two dimensional notation for the stencils allows to visualize the application of multi-diagonal matrices so Eq.(8) corresponds to:

$$
\begin{aligned}
& \frac{1}{6 h}\left(-20 u_{i_{1}, i_{2}}+4 u_{i_{1}+1, i_{2}}+4 u_{i_{1}-1, i_{2}}+4 u_{i_{1}, i_{2}+1}+4 u_{i_{1}, i_{2}-1}+u_{i_{1}+1, i_{2}+1}+u_{i_{1}-1, i_{2}+1}\right. \\
& \left.\quad+u_{i_{1}+1, i_{2}-1}+u_{i_{1}-1, i_{2}-1}\right)=\frac{1}{12}\left(8 v_{i_{1}, i_{2}}+v_{i_{1}+1, i_{2}}+v_{i_{1}-1, i_{2}}+v_{i_{1}, i_{2}+1}+v_{i_{1}, i_{2}-1}\right)
\end{aligned}
$$

for all $i_{1}, i_{2}$.

We apply a data structure and an algorithm similar to [65] where the author restrains to the classical second order Poisson solver. Rather than applying the "Full Approximation Scheme" which consists in starting the approximation algorithm at the coarser levels before doing the V-cycles, we 


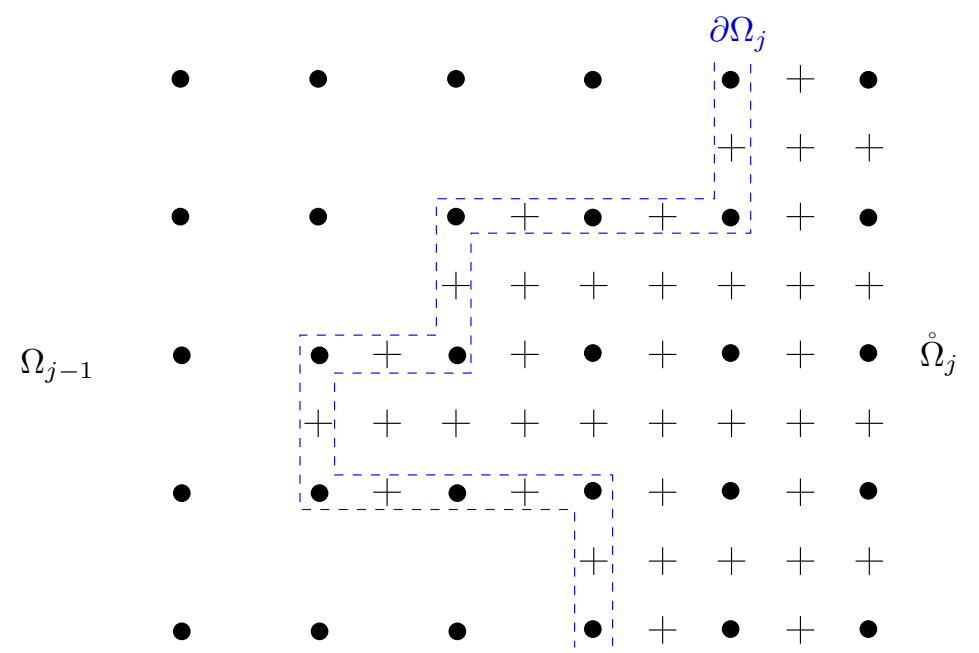

Figure 1: Example of domain $\Omega_{j}$ divided between its boundary $\partial \Omega_{j}$ and its interior $\AA_{j}$.

initiate the algorithm in a "Residual-based" way with the previous time step result as a first guess.

In the following, we assume the domain $[-L / 2, L / 2]^{2}$ to be paved with different continuous domains $\bar{\Omega}_{j}$ such that $\bar{\Omega}_{j+1} \subset \bar{\Omega}_{j}$. For $j \geq 0$, the domain $\Omega_{j}$ is discretized with a space step $h_{j}=2^{-j-1} L$. The structure described in Tab. 1 generates such a mesh. We also consider the boundary $\partial \Omega_{j}$ of the domain $\Omega_{j}$ as composed of the points with missing direct neighboring points in $\Omega_{j}$ in one of the four directions or in diagonal. We note $\Omega_{j}=\Omega_{j} \backslash \partial \Omega_{j}$ (see Fig.1 for an illustration).

We will solve Eq. (8) on all points of $\Omega_{j}$ while the points of $\partial \Omega_{j}$ will be interpolated from $\Omega_{j-1}$ providing boundary conditions for $\Omega_{j}$.

The resulting algorithm is of multigrid type with values of $v$ known at the points of the mesh $\cup_{j=0}^{j_{\max }} \Omega_{j}$ and values of $u$ to be determined at these points.

In this algorithm, the residual is computed at the finest level: if $\mathbf{x} \in \AA_{j}$ and $\mathbf{x} \notin \Omega_{j+1}$ then the residual at $\mathbf{x}=\left(i_{1} h_{j}, i_{2} h_{j}\right)$ is

$$
r(\mathbf{x})=\frac{1}{12}\left[\begin{array}{lll}
0 & 1 & 0 \\
1 & 8 & 1 \\
0 & 1 & 0
\end{array}\right] v_{i_{1}, i_{2}}-\frac{1}{6 h_{j}^{2}}\left[\begin{array}{ccc}
1 & 4 & 1 \\
4 & -20 & 4 \\
1 & 4 & 1
\end{array}\right] u_{i_{1}, i_{2}} .
$$

At the end of the Gauss-Seidel iterations at level $j$ (two iterations in our 
code), the residual $r(\mathbf{x})$ is restricted to provide the residual at point $\mathbf{x}$ and level $j-1$.

Conversely, during the prolongation process, the boundary $\partial \Omega_{j}$ is interpolated from level $j-1$. This interpolation has to be sixth order accurate because the Dirichlet boundary conditions require a $C^{6}$ continuity to ensure the fourth order accuracy of the Collatz solver (Eq.(8)) on $\Omega_{j}$.

Nevertheless the restriction of the residual and of the prolongation of the "residual solution" are the classical second order multigrid restriction and prolongation used in [71]: $\frac{1}{4}\left[\begin{array}{lll}1 & 2 & 1\end{array}\right]$ and $\frac{1}{2}\left[\begin{array}{lll}1 & 2 & 1\end{array}\right]$ respectively. The whole algorithm is detailed in Appendix A.

It is possible to extend this scheme to solve

$$
\left(\alpha I d-\beta \nabla^{2}\right) u=v+\nabla^{2} w
$$

where $\alpha \beta \geq 0, \alpha \neq 0$ or $\beta \neq 0, v$ and $w$ are known and $u$ is the unknown function with the fourth order accuracy approximation:

$$
\begin{aligned}
& \left(\frac{\alpha}{12}\left[\begin{array}{lll}
0 & 1 & 0 \\
1 & 8 & 1 \\
0 & 1 & 0
\end{array}\right]-\frac{\beta}{6 h^{2}}\left[\begin{array}{ccc}
1 & 4 & 1 \\
4 & -20 & 4 \\
1 & 4 & 1
\end{array}\right]\right) u \\
& =\frac{1}{12}\left[\begin{array}{lll}
0 & 1 & 0 \\
1 & 8 & 1 \\
0 & 1 & 0
\end{array}\right] v+\frac{1}{6 h^{2}}\left[\begin{array}{ccc}
1 & 4 & 1 \\
4 & -20 & 4 \\
1 & 4 & 1
\end{array}\right] w .
\end{aligned}
$$

This general formulation may be specialized to

- $\alpha=0, \beta=-1, w=0$ for the Poisson equation $\nabla^{2} u=v$,

- $\alpha=1, \beta=\nu \Delta t, w=0$ for the implicit Euler integration of the Heat Equation $\partial_{t} u=\nu \nabla^{2} u$, useful in the resistive case,

- $\alpha=1, \beta=d_{e}^{2}, v=0$ and $w=-F$ for the computation of $J_{z}$ given by the equation $\left(I d-d_{e}^{2} \nabla^{2}\right) J_{z}=-\nabla^{2} F$.

The convergence of this kind of multi-grid algorithms is studied in [71]. It allows an exponential convergence to the solution with a rate independent of the finest space step $h$. From our experience, the shape of the domains $\Omega_{j}$ may have an influence on the convergence rate only in the most pathological cases. Most importantly, this solver guarantees a fourth order accuracy as confirmed by Fig.2 left. 


\subsection{CFL condition for the RMHD equations}

In the case $\eta \neq 0$, the viscous term has to be treated implicitly, otherwise a penalizing $\Delta t \leq \Delta x^{2}$ stability condition would appear. Then, the choice of a particular IMEX scheme (Implicit-Explicit scheme [64]) largely influences the stability conditions.

In order to derive a proper CFL condition adapted to the RMHD equations Eq.(1), (2) and (3) for $\eta=\nu=0$, we apply a routine von Neumann stability analysis [75] detailed in Appendix B.

From this analysis we derive the stability condition:

$$
\Delta t \leq C \frac{\Delta x}{\|\boldsymbol{\nabla} \varphi\|_{\infty}+\frac{\rho_{s}^{2} \Delta x^{2}}{\Delta x^{2}+d_{e}^{2}}\|\nabla U\|_{\infty}+\left(\frac{2 \rho_{s}^{2}+\Delta x^{2}}{\Delta x^{2}+d_{e}^{2}}\right)^{1 / 2}\|\nabla F\|_{\infty}},
$$

where $\|\cdot\|_{\infty}=\left\||\cdot|_{\ell^{1}}\right\|_{L^{\infty}(\Omega)}$.

In the case $\rho_{s} \neq 0$ and $d_{e}=0$ (which is however not of interest to the reconnection problems we consider here, which require either $d_{e} \neq 0$ or $\eta \neq 0$ ), we obtain the condition

$$
\Delta t \leq C \frac{\Delta x^{2}}{\sqrt{2} \rho_{s}\|\nabla F\|_{\infty}},
$$

which is particularly restrictive.

In the case $\rho_{s}=0$ and $d_{e} \neq 0$, we obtain

$$
\Delta t \leq C \frac{\left(\Delta x^{2}+d_{e}^{2}\right)^{1 / 2} \Delta x}{\|\boldsymbol{\nabla} \varphi\|_{\infty}\left(\Delta x^{2}+d_{e}^{2}\right)^{1 / 2}+\|\boldsymbol{\nabla} F\|_{\infty} \Delta x},
$$

where $\|\cdot\|_{\infty}=\left\||\cdot|_{\ell^{1}}\right\|_{L^{\infty}(\Omega)}$. This inequality is implied by the simpler condition

$$
\Delta t \leq C \frac{d_{e} \Delta x}{\|\boldsymbol{\nabla} \varphi\|_{\infty} d_{e}+\|\boldsymbol{\nabla} F\|_{\infty} \Delta x} .
$$

The CFL condition provided by Eqs.(13-14) allows much larger time steps at the beginning of the numerical experiment than later, when the singularity is developed. Having implemented it at each time step allows a substantial economy in the computation and also discards the numerical instabilities which appear when using any unfit time step. 
We finally note that, by using Eqs.(5) and (B.1), Eq.(13) can be rewritten in terms of the vector components as

$$
\Delta t \leq C \frac{\left(\Delta x^{2}+d_{e}^{2}\right)^{1 / 2} \Delta x}{\|\boldsymbol{u}\|_{\infty}\left(\Delta x^{2}+d_{e}^{2}\right)^{1 / 2}+\left\|\boldsymbol{B}_{\perp}-d_{e}^{2} \nabla^{2} \boldsymbol{B}_{\perp}\right\|_{\infty} \Delta x} .
$$

This inequality is in turn implied by the simpler condition:

$$
\Delta t \leq C \frac{d_{e} \Delta x}{\|\boldsymbol{u}\|_{\infty} d_{e}+\left\|\boldsymbol{B}_{\perp}\right\|_{\infty} \Delta x}
$$

\section{Physical model for reconnection instabilities}

We recall that Eqs.(1-3) describe the dynamics in the Alfvénic frequency range of an MHD plasma in a strong guide magnetic field, whose dominant, uniform component is oriented along $z$. Eq.(1) corresponds to the $z$-component of the magnetic induction equation obtained from the generalized Ohm's law in which resistivity $(\eta)$, electron inertia effects (related to the ion skin depth $d_{e}$ ) and electron temperature effects (related to the ionsound Larmor radius $\rho_{s}$ ) have been retained; Eq.(2) is the $z$-component of the plasma vorticity equation, which includes ion-ion viscosity $(\nu)$. As already mentioned (Sec.2.1) $\varphi$ and $\psi$ are the stream functions respectively related to the fluid $\boldsymbol{E} \times \boldsymbol{B}$-drift plasma velocity, $\boldsymbol{u}=\boldsymbol{e}_{z} \times \boldsymbol{\nabla} \varphi$, and to the in-plane magnetic field, $\boldsymbol{B}_{\perp}=\boldsymbol{\nabla} \psi \times \boldsymbol{e}_{z}$; they are in turn related by Eqs.(3) to the $z$-component of the electron canonical momentum $F$ and to the $z$-component of the fluid vorticity $U$. Further details about the derivation and meaning of the equations can be found for example in Refs.[18, 57]. Eqs.(1-3) are written in non-dimensional units, with density and magnetic field expressed in units of their reference values $n_{0}$ and $B_{0}$, lengths normalized to the equilibrium shear length $a$, and velocities to the Alfvén velocity $c_{A} \equiv B_{0} / \sqrt{4 \pi n_{0} m_{i}}$, with $m_{i}$ the ion mass. The parameters $\eta, d_{e}, \rho_{s}$ and $\nu$ are therefore dimensionless with respect to their appropriate normalizations.

Note that resistive reconnection, especially in space-plasma literature, often refers to the dimensionless Lundquist number $S \equiv c_{A} a / \eta_{m}$, defined as the inverse of the magnetic diffusity $\eta_{m}$, normalized to the characteristic Alfvén time measured with respect to the scale $a$. Because of the normalization of Eq.(1) the dimensionless resistivity $\eta$ coincides with the so-normalized magnetic diffusivity, so that it can be read $\eta \equiv S^{-1}$.

Concerning the normalization of lengths we finally recall that, due to the separation of scales which can be encountered in different reconnection 
problems, it is generally important to distinguish between $L_{x}, L_{y}$ (dimensions of the simulation box), $a_{c s}$ and $L_{c s}$ (width and length, respectively, of a generic current sheet). We name the latter respectively $a$ and $L$ when referred to the equilibrium quantities. Appropriate rescaling arguments should be therefore considered when applying the tearing mode theory to a generic current sheet of aspect ratio $L_{c s} / a_{c s}$, such as that generated by primary instabilities.

\subsection{Reconnection instability and $\Delta^{\prime}$ parameter}

By choosing a magnetic equilibrium profile $\psi_{e q}(x)$, sheared with respect to the $x$ variable and not dependent on $y$, we are interested in studying reconnection instabilities which develop by exciting the perturbations of the kind $f(x) e^{i\left(k_{y} y-\omega t\right)} e^{\gamma t}$, with $k_{y}, \omega, \gamma$ real parameters, the latter thus expressing the growth rate of the tearing-type instability. This instability induces the formation of the characteristic magnetic island structures, linearly developing inside a reconnection layer of characteristic width $\delta$, centered around the resonant position. For a locally monotone $\psi_{e q}^{\prime}(x)$, the latter corresponds to the value of $x$ where $\psi_{e q}^{\prime}(x)=0$. The study of the reconnection instabilities leads to the eigenvalue problem related to the linearized Eqs.(1-3), which is solved as a boundary layer problem [76]: an outer solution $\psi_{\text {out }}$, which depends only on the shape of $\psi_{e q}(x)$ and on the wave-number $k_{y}$, is obtained in the "outer" region (i.e. for $x / a \sim 1$ ), in which the ideal MHD limit is assumed $\left(d_{e}=\rho_{s}=\eta=\nu=0\right)$; $\psi_{\text {out }}$ is then exploited as "boundary condition" for the inner solution, to which it is matched, and that is sought for the system in an inner layer, close $x \rightarrow 0$, where non-ideal MHD effects result non negligible. Depending on the shape of the equilibrium profile $\psi_{e q}(x / a)$, which varies over the characteristic length $a$, different wavenumbers $k_{y}$ may result unstable. The instability condition can be related to the so-called $\Delta^{\prime}$ parameter, formally defined [76] as the derivative of the logarithmic jump of the solution $\psi_{\text {out }}$ as it approaches the inner layer,

$$
\Delta^{\prime} \equiv \frac{\psi_{\text {out }}^{\prime}\left(0^{+}\right)-\psi_{\text {out }}^{\prime}\left(0^{-}\right)}{\psi_{\text {out }}(0)}
$$

This parameter, which can be shown (see, e.g., Ref.[1], Sec.4.1) to be proportional to the amount of free magnetic energy that can be transferred from the ideal region into the reconnection layer during the linear growth of the

resistive instability, must be positive in order for a given tearing-type mode 
(i.e. a fixed $k_{y}$ ) to be unstable. This condition fixes, for an unstable mode with $m=k_{y} L /(2 \pi)$, the maximum number of oscillations $m_{\max }$ on a current sheet with length $L$. This will be discussed in the simulation examples to be considered next.

Furthermore, depending on the mechanism violating the ideal Ohm's law, here expressed by the generic parameter $\varepsilon$, it can be shown that the relative magnitude of $\Delta^{\prime}\left(k_{y} a\right) \delta(\varepsilon)$ with respect to unity identifies different regimes of the reconnection instabilities, where the growth rate $\gamma$ and the layer width $\delta$ take different scalings with respect to $\varepsilon$ (see $[77,78]$ for the resistive regime and $[79,32]$ for the collisionless one). Following the standard terminology we name these three regimes "small- $\Delta^{\prime}$ " (SD), for $\Delta^{\prime} \delta \ll 1$, "large- $\Delta^{\prime}$ " (LD), for $\Delta^{\prime} \delta \gg 1$, and "fastest growing mode", for $\Delta^{\prime} \delta \simeq 1$. The latter corresponds to the maximally growing wave-number $k_{M}$ (at fixed $\varepsilon$ ) in a continuum wave-length spectrum of unstable modes. It can be shown [18] that both the wave-number and the growth rate of the fastest growing mode are, in general, function of $\varepsilon$ only, $k_{M}(\varepsilon)$ and $\gamma_{M}(\varepsilon)$. In a continuum wavelength spectrum the asymptotic limits of the LD and SD regimes correspond respectively to $k_{y} \ll k_{M}(\varepsilon)$ and $k_{y} \gg k_{M}(\varepsilon)$ [80]. Recent discussions on the transition among the three regimes can be found in Refs.[18, 31, 80].

\subsection{Hamiltonian limit}

The low-collision regime which characterizes most astrophysical and fusion plasmas corresponds to a parameter range in which $\eta$ and $\nu$ are often much smaller than both $d_{e}^{2}$ and $\rho_{s}^{2}$. Even if this does not generally imply that the effects of $\eta$ and $\nu$ on the reconnection rate and on the nonlinear dynamics can be totally neglected (see [18]), in the numerical applications which follow we focus on the completely collisionless, inviscid limit $(\eta=0$ and $\nu=0$ ) of Eqs.(1-3), in which reconnection is possible whenever $d_{e} \neq 0$. This limit is particularly interesting because, on the one hand, the stiffness of the corresponding equations makes it particularly challenging from a computational point of view, and therefore compelling to test the AMR code. On the other hand, its Hamiltonian character implies the existence of an infinity of integral constants of motion besides the total energy [81, 82] and also grants the Lagrangian conservation of local quantities dragged by the fluid flow $\boldsymbol{u}[25,81]$. These can be used to check the accuracy and conservations of the algorithm during the numerical integration.

We recall in particular that the conservation of the total energy follows 
from Eqs.(1-3) at $\eta=\nu=0$ :

$$
E \equiv \iint\left(|\nabla \psi|^{2}+d_{e}^{2}\left|\nabla^{2} \psi\right|^{2}+|\nabla \varphi|^{2}+\rho_{s}^{2}\left|\nabla^{2} \varphi\right|^{2}\right) d x d y
$$

Then, for $\rho_{s} \neq 0[81,25]$, the two following families of Casimir's integrals commute with the Hamiltonian,

$$
C_{+} \equiv \iint f_{+}\left(F+d_{e} \rho_{s} U\right) d x d y, \quad C_{-} \equiv \iint f_{-}\left(F-d_{e} \rho_{s} U\right) d x d y,
$$

with $f_{+}$and $f_{-}$arbitrary functions. In this regime Eqs.(1-2) can be manipulated to express the two following Lagrangian invariances,

$$
\frac{d_{+}}{d t}\left(F+d_{e} \rho_{s} U\right)=0, \quad \frac{d_{-}}{d t}\left(F-d_{e} \rho_{s} U\right)=0,
$$

with respect to the flows

$$
\frac{d_{ \pm}}{d t} \equiv \frac{\partial}{\partial t}+\left[\varphi \pm\left(\rho_{s} / d_{e}\right) \psi, \cdot\right]
$$

The "cold electron" $\rho_{s}=0$ case is instead singular with respect to the corresponding limit of Eqs.(19-21). The two families of Casimir's invariants are replaced by

$$
C_{1} \equiv \iint f_{1}(F) d x d y, \quad C_{2} \equiv \iint U f_{2}(F) d x d y
$$

with $f_{1}$ and $f_{2}$ arbitrary functions. In this case only $F$ results a Lagrangian invariant advected by the flow generated by $\varphi$,

$$
\frac{d}{d t} F=0
$$

where $d / d t \equiv \partial_{t}+[\varphi, \cdot]$.

\subsection{Linear analysis in the Hamiltonian limit}

In the RMHD Hamiltonian limit, the stable, normal modes propagating in a homogeneous plasma with a wave vector of modulus $k$ having a component $k_{\|}$along a uniform, in-plane magnetic field component, correspond to the dispersive, kinetic-Alfvén waves. Their dispersion relation is

$$
\omega^{2}=k_{\|}^{2} \frac{1+k^{2} \rho_{s}^{2}}{1+k^{2} d_{e}^{2}} .
$$


In the "cold" $\rho_{s}=0$ limit they display the same kind of stiffness of whistler waves in 2D incompressible EMHD (see [57] for a comparison of the 2D RMHD and EMHD models), having an identical dispersion relation but for the substitution $k_{\|}^{2} \rightarrow k_{\|} k$. In the "warm" $\rho_{s} \neq 0$ limit, they describe fast dispersive waves - see e.g. [83] for a recent discussion about these modes and for their implications for implicit solvers of the RMHD set.

As discussed in Sec.3.1, the unstable tearing mode we are interested in develops instead when the in-plane component of the magnetic field is sheared, with a locally linear dependence near a singular position. As already recalled in the same Section, the corresponding eigenvalue problem can be analytically solved by relying on the asymptotic analysis, here valid when $d_{e} / a \rightarrow 0$.

In the "cold" $\rho_{s}=0$ limit the linear dispersion relation so obtained assumes, by restoring dimensional quantities, the following implicit form $[32,19]$,

$$
\frac{\gamma \tau_{A}}{k a}=-\frac{\pi}{a \Delta^{\prime}(k a)}\left(\frac{\gamma \tau_{A} a}{4 k d_{e}^{2}}\right)^{\frac{3}{2}} \frac{\Gamma((Q-1) / 4)}{\Gamma((Q+5) / 4)}, \quad Q \equiv \frac{\gamma \tau_{A} a}{k d_{e}^{2}}
$$

with $\tau_{A} \equiv c_{A} / a$ being the reference Alfvén time and $\Gamma$ noting Euler's Gammafunction. The scaling in the different $\Delta^{\prime}$ regimes can be estimated from this expression (see Appendix C of Ref.[31] for a brief review and discussion of this technique).

In particular, the scalings of the maximally growing mode satisfying $\Delta^{\prime}\left(k_{M} a\right) \delta\left(k_{M}, d_{e}\right) \simeq 1$ in the asymptotic limit of a sufficiently large aspect ratio current sheet, generally depend on the choice of the equilibrium profile. For the equilibrium choice considered in the following numerical examples (cf. Eq.(36) next) these scalings would read [18]:

$$
k_{M} a \sim\left(\frac{d_{e}}{a}\right)^{\frac{1}{2}}, \quad \gamma_{M} \tau_{A} \simeq\left(\frac{d_{e}}{a}\right)^{\frac{3}{2}}, \quad \frac{\delta_{M}}{a} \sim \frac{d_{e}}{a} .
$$

When applying Eq. (25) to a continuum wave-length spectrum, the LD $\left(\Delta^{\prime} \delta \gg\right.$ $1)$ and $\mathrm{SD}\left(\Delta^{\prime} \delta \ll 1\right)$ conditions would imply a restriction to the wavelength ranges $k_{y} a \ll\left(d_{e} / a\right)^{1 / 2}$ and $k_{y} a \gg\left(d_{e} / a\right)^{1 / 2}$ respectively. In these regimes Eq.(25) can be approximated as $[17,79]$

$$
\gamma_{L D} \tau_{A} \simeq k d_{e}, \quad \gamma_{S D} \tau_{A} \simeq\left(\frac{\Gamma(1 / 4)}{2 \pi \Gamma(3 / 4)}\right)^{2} \frac{k d_{e}^{3}}{a^{2}}\left(\Delta^{\prime} a\right)^{2} .
$$


The respective width of the current layers generated by the reconnecting instability during the linear phase are estimated to be $[17,79]$

$$
\frac{\delta_{L D}}{a} \sim \frac{d_{e}}{a}, \quad \frac{\delta_{S D}}{a} \sim \frac{\Delta^{\prime} d_{e}}{a} .
$$

For $\rho_{s} \neq 0$, instead, the analytical expression replacing (25) in the "warm" Hamiltonian limit is much more cumbersome [84]. Moreover, its simplified rewriting in some restricted wave-length ranges [32] gives estimations of the dispersion relations, whose validity in the parameter space is a priori not so easily recognizable [24]. Therefore, in this "warm" case, as well as, more generally, when the value of $d_{e} / a$ is not sufficiently small, both for null and non-null values of $\rho_{s}$, it is better to rely on a numerical solution of the eigenvalue problem in order to compute the growth rates of the reconnecting instabilities.

To this purpose, we numerically compute the growth rate of each unstable mode of the type $f(x) e^{i k y} e^{\gamma t}$ in a similar way as in the CFL analysis Appendix B by considering:

$$
\begin{array}{r}
\psi(x, y)=\psi_{e q}(x)+\tilde{\psi}(x) e^{i k y} e^{\gamma t}, \\
F(x, y)=F_{e q}(x)+\tilde{f}(x) e^{i k y} e^{\gamma t} \\
\varphi(x, y)=\tilde{\varphi}(x) e^{i k y} e^{\gamma t} \\
U(x, y)=\tilde{u}(x) e^{i k y} e^{\gamma t}
\end{array}
$$

and by solving the eigen-value problem below (with normalized quantities as in Eqs.(1-3))

$$
\gamma^{2} \tilde{\varphi}=-k^{2} A^{-1} B C^{-1} D \tilde{\varphi}
$$

with

$$
\begin{array}{r}
A=\partial_{x}^{2}-k^{2}, \quad B=\psi_{e q}^{\prime} \partial_{x}^{2}-k^{2} \psi_{e q}^{\prime}-\psi_{e q}^{\prime \prime \prime}, \\
C=1+k^{2} d_{e}^{2}-d_{e}^{2} \partial_{x}^{2}, \quad D=F_{e q}^{\prime}+\rho_{s}^{2} k^{2} \psi_{e q}^{\prime}-\rho_{s}^{2} \psi_{e q}^{\prime} \partial_{x}^{2},
\end{array}
$$

while using a non regular grid on the $x$-axis in order to refine the approximation near zero at the singularity.

\section{Numerical experiments in the Hamiltonian limit}

In order to test the performances of the code we have run numerical simulations of collision-less reconnecting instabilities on a square simulation 
box of dimension $\left[-L_{x} / 2, L_{x} / 2\right] \times\left[-L_{y} / 2, L_{y} / 2\right]$, with $a=0.5$ and $L_{x}=$ $L_{y}=2 \pi$ or $a=1$ and $L_{x}=L_{y}=4 \pi$, for different values of the ratio $\rho_{s} / d_{e}$ and for the equilibrium profile [85]

$$
\psi_{e q}(x)=-\frac{1}{2} \frac{1}{\cosh ^{2}(x / a)}, \quad \Delta^{\prime} a=2\left(\frac{-\left(k_{y} a\right)^{4}+2\left(k_{y} a\right)^{2}+15}{\left(k_{y} a\right)^{2} \sqrt{4+\left(k_{y} a\right)^{2}}}\right) .
$$

This has been chosen in place of the more customary Harris-pinch profile $[86], B_{y}^{0}(x)=B_{y}^{0} \tanh (x / a)$, whose instability parameter is $\Delta^{\prime} a=2\left(1 /\left(k_{y} a\right)-\right.$ $\left.k_{y} a\right)$, because of the numerical constraint given by the periodic boundary conditions. Although equilibrium (36) formally has three null lines, respectively at $x=0$ and at $x= \pm \infty$, only the former is of practical relevance due to the vanishing current density at $x= \pm \infty$. This profile has been much exploited for numerical studies of "fast" reconnection in the LD regime due to the large values of the $\Delta^{\prime}$ parameter that can be achieved with relatively small values of the ratio $L_{y} / a$.

The condition $\Delta^{\prime} a>0$ for the equilibrium described by Eqs.(36) corresponds to unstable modes satisfying $0<k_{y} a<\sqrt{5}$. This condition translates on a constraint about the number of oscillations $m \equiv k_{y} L_{y} /(2 \pi)$ of the modes which can be destabilized in a simulation box with finite size $L_{y}$, which reads

$$
m \leq \operatorname{int}\left(\frac{\sqrt{5}}{2 \pi} L_{y}\right)
$$

Finally note that, by construction, the aspect ratio of the equilibrium current sheet subject to the first reconnection process is $L / a=L_{y} / a$. In the following the adjective "classical" is meant for usual tearing-type instabilities with $a$ as macroscopic length, in contraposition to configurations in which $L / a \gg 1$ and the macroscopic reference length is $L=L_{y}$ (case, for instance, of the "ideal" tearing, of the plasmoid instability or of Sweet-Parker reconnection).

In Sec.4.1 we discuss an example of simulation in the "cold" regime $\left(\rho_{s}=0\right)$, which emphasizes the usefulness of the AMR algorithm to follow the spatial filamentation and turbulent dynamics typical of this case, and highlights the influence of the latter in the loss of integral conservations (e.g., energy) and in the breaking of spatial symmetries of the linear eigenfunctions. The choice of the non-ideal parameters is the same as previous simulations available in literature, to which the result will be compared, although mainly from a qualitative point of view: due to a peculiar choice of 
the initialization of the perturbation, the example here presented aims indeed to highlight some delicate issues about the interpretation of the appearance of "plasmoids" during the nonlinear stage of a primary reconnecting mode.

In Sec.4.2 we provide an example of linear validation of the code in the warm $\rho_{s} \neq 0$ regime, by comparing some growth rates, measured after integration of the RMHD set, with the values reported in previous studies available in literature, as well as with the values obtained by numerical solution of the eigenvalue problem (Sec.3.3). We then discuss an example of nonlinear simulation in which the ratio $\rho_{s} / d_{e}>1$ makes the turbulent dynamics be replaced by the laminar evolution first evidenced in Refs. [25, 27]. The numerical results here presented for this explorative simulation indicate however that, also in the late nonlinear stage of this warm regime, fluid instabilities, which at the best of the knowledge of the authors have never been evidenced before in this parameter range, may develop.

\subsection{Test case (1): "classical" tearing with $\rho_{s}=0$}

As a first benchmark test we have run simulations using the equilibrium of Eq.(36) for $d_{e}=0.3 a$ and $\rho_{s}=0$. This is the case studied in Ref.[20], in which the vorticity and current sheets, developed along the neutral line by the primary reconnecting mode, become rapidly unstable to secondary fluid instabilities, especially of KH-type. This makes the numerical simulation of this nonlinear dynamics particularly challenging. The large value of $d_{e} / a$ also implies relatively large growth rates, approaching values comparable to the Alfvénic time scale $\tau_{A}^{-1}$.

Regarding the AMR parameters (cf. Sec.2.4) the refinement criterion

$$
w_{\lambda} \geq 2^{\alpha j-1} \epsilon
$$

is here applied with $\alpha=1.3$ and $\epsilon=1.125 e-5$ (empirically defined) to both the functions $F$ and $U$ through the value $w_{\lambda}=\sum_{\kappa \in\{-1,0,1\}^{2}} v_{\lambda+\kappa}$ (cf. Eq.(7)), where

$$
v_{\lambda}=\sqrt{d_{\lambda}(F)^{2}+d_{\lambda}(U)^{2}}
$$

and $d_{\lambda}$ is the residual of the second order interpolation performed from the coarser level (actual value at level $j$ minus value interpolated from level $j-1)$. Moreover we take $j_{\text {lim }}=12$. This choice of $\alpha$ and $\epsilon$ allows to run this experiment for $t \in[0,30]$ in a few hours on a 8-cores computer node.

First we validate the fourth order accuracy for the AMR Poisson Solver (Sec.2.5) for Eqs.(3) and the third order accuracy of the whole scheme for 
Eqs. $(1,2)$. The advection terms in Eqs. $(1,2)$ are computed with an upwind third order scheme.

For the test of the Poisson solver, we solve $\nabla^{2} u=\sin (6 \pi x) \sin (6 \pi y)$ on a grid containing three different levels of refinement and we double the accuracy six times to measure the convergence (Fig. 2 left). For the whole scheme, we run the "classical" tearing test case in the linear regime of the growth of reconnection, we stop at $t=10$ and we compute the error on $F(t=10)$ by comparing to a reference solution on the finest mesh containing four different levels of refinement. We sequentially double mesh sizes and observe the third order of convergence (Fig. 2 right).
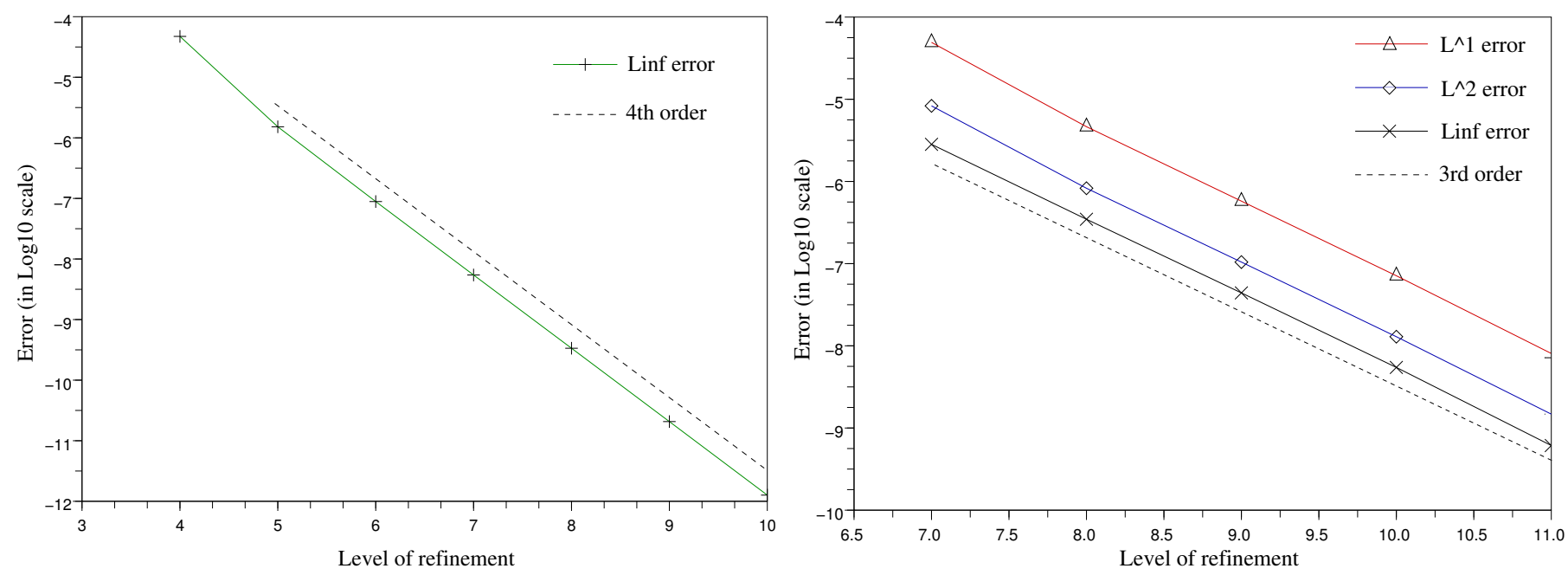

Figure 2: Test of convergence on non-uniform grids by means of accuracy doubling. The error is represented in log-scale amplitude. $L^{1}, L^{2}$ and $\operatorname{Linf}$ label the errors measured with respect to the norms $\|\cdot\|_{L^{1}},\|\cdot\|_{L^{2}}$ and $\|\cdot\|_{L^{\infty}}$ norms, and " 4 th order" and "3rd order" mean the functions $f(h)=C h^{4}$ and $f(h)=C h^{3}$ respectively. Left: test of the AMR Collatz solver. Right: test of the whole scheme, error on $F(t=10)$.

The value of the non-ideal parameter $\varepsilon=d_{e} / a$ allowing reconnection has been chosen for comparison with the simulations run in Refs.[20, 87]. However, the different choice of the box dimensions, $L_{x}=2 L_{y}=4 \pi a$ in the latter case, in contrast to the $L_{x}=L_{y}=4 \pi a$ case considered here, implies important differences on the number of magnetic islands (i.e. the mode number $m$ ) generated by the most unstable mode: for $L_{y}=2 \pi a$, just the modes $m=1$ and $m=2$ result unstable, respectively corresponding to $k_{y} a=1$ and $k_{y} a=2$; for the case $L_{y}=4 \pi a$, instead, the modes $m=1,2,3$ 
and 4 are unstable, respectively corresponding to $k_{y} a=0.5,1,1.5$ and 2 .

Moreover, the relatively large value chosen for $d_{e} / a$ makes the linear analytical estimation (25) not accurate (cf. Fig.1 of Ref.[58]), and therefore also Eqs.(26-28) result inappropriate.

The values of the growth rate $\gamma_{e i g}$ of the unstable wave-vectors $k_{y} a$, obtained by numerical solution of the eigenvalue problem as described at the end of Sec.3.3, are compared in Table 2, in which they are written together with the corresponding values of the normalized $\Delta^{\prime}$ parameter.

Table 2: Growth rates and values of the $\Delta^{\prime}$ parameter as a function of the normalized wavevector $k_{y} a$, for the mode numbers unstable when $L_{y}=2 \pi a$ and $L_{y}=4 \pi a$ respectively.

\begin{tabular}{|c||c|c|c|c|}
\hline$m$ for $L_{y}=2 \pi a$ & - & 1 & - & 2 \\
\hline$m$ for $L_{y}=4 \pi a$ & 1 & 2 & 3 & 4 \\
\hline$k_{y} a$ & 0.5 & 1 & 1.5 & 2 \\
\hline$\Delta^{\prime}\left(k_{y} a\right)$ & 59.90 & 14.31 & 2.20 & 1.23 \\
\hline$\gamma_{e i g}\left(k_{y} a\right) \tau_{A}$ & 0.103 & 0.125 & 0.070 & 0.008 \\
\hline
\end{tabular}

As shown in Fig.3, where the growth of the Fourier transform of the unstable modes (averaged with respect to the $x$-variable) is represented in time, the simulation we have run has been initialized by perturbing the equilibrium of Eq.(36) with a function of the kind

$$
\tilde{\psi}(x, y)=\epsilon \cos (y / 2)
$$

thus exciting only the $m=1$ mode with an amplitude $\epsilon=10^{-5}$. This mode is responsible of the growth of the first magnetic island, well visible in Fig.4 since time $t=25 \tau_{A}$. As first evidenced in Ref.[20] and re-discussed in Ref.[57], the nonlinear dynamics of the current layers is driven in this regime by the evolution of the vorticity sheets, which advect the field $J_{z}$ : this induces the appearance of secondary fluid instabilities both of Kelvin-Helmholtz-type, which start affecting the current sheets inside the island since $t=26.6$, and of "interchange" type (i.e., Rayleigh-Taylor/Richtmyer-Meshkov instabilities), related to the characteristic mushroom patterns visible since $t=26.6$ and to the subsequent perturbations on their surface, shown since $t=27.2$ (Fig.5). This dynamics rapidly leads to a turbulent regime inside the magnetic island, which is associated to the generation of increasingly smaller spatial scales, as evidenced by the level of refinement necessary to follow this dynamics, which has been quantified in Figs.4-5 (second row of each figure) and in Fig.6. It 
is however worth remarking that, even before the onset of turbulence, the increase of the grid refinement is required by the spatial filamentation, which is an intrinsic feature of this Hamiltonian system: this is exemplified by the progressive thinning of the current sheet $J_{z}$ along the neutral line $[17,19]$, whose evolution in time at $y=3 L_{y} / 8=3 \pi / 4$ is shown in Fig.7.

The nonlinear cascade towards small spatial scales associated to this turbulent regime leads to a rapid deterioration of the integral conservations by numerical dissipation, which can be delayed only at the price of an enormous computational effort - we recall indeed that the increase in the grid refinement couples with more severe CFL conditions, as it is expressed by Eqs.(13-14): having fixed, in this numerical example, the maximum refinement to $h_{\text {lim }}=2^{-12} L$, the total energy, perfectly conserved according to Eq.(18) until $t \simeq 27$, looses about $14 \%$ of its initial value by time $t=30$ and drops down to $65 \%$ of its initial value by time $t=50$ (see Fig.8).

Finally note that the AMR algorithm we have discussed allows to preserve the spatial symmetry of the fields also in the nonlinear stage, in principle as long as the grid refinement can accurately describe the generation of small spatial scales: during the nonlinear stage, as soon as the current sheets moving inside the magnetic island from the two $X$-points "collide" and bend (Fig.4, at $t=26.6$ ), the distinct $x \leftrightarrow-x$ and $y \leftrightarrow-y$ symmetry invariances of the linear eignemodes merge into a central symmetry by rotation around the magnetic island's $O$-point (i.e., an invariance with respect to $(x, y) \leftrightarrow(-x,-y)$ transformations). This central symmetry is however lost as soon as numerical errors at grid scale cumulate (cf. Fig.1 of Ref.[20]). In the simulation we have presented we see that it is only when the grid refinement becomes appreciably insufficient to grant energy conservation (cf. Fig. 8 for $t \simeq 30$ ) that the central symmetry is broken (Fig.5, $t=32.4$ ).

We conclude this section with a brief comparison with the simulations of Refs. $[20,87]$ and a comment about the particular choice of initialization expressed by Eq.(40). We first note that the time scales of the simulations of Refs. $[20,87]$ and those here discussed are appreciably different mainly because of differences due to initialization of the perturbations, which implies quite different transient times (also because of the role played by the spectral-like filters, used in the simulations of Refs.[20, 87] to prevent aliasing problems). Then, as evidenced in Table 2 , the $m=1$ mode excited in Ref.[20] corresponds to $k_{y} a=1$, whereas, in the simulations here reported, it corresponds to $k_{y} a=0.5$. However, while the growth rates of $k_{y} a=1$ and $k_{y} a=0.5$ are comparable, the $m=2$ mode has a negligible growth rate in 
the case studied in Ref.[20], whereas in the simulations shown in Figs.(4-5) is dominant and is responsible of the appearance of the second island at the edges of the simulation box (well visible at $t=28.4$ in Fig.5). Therefore, as evidenced by the Fourier spectrum displayed in Fig.3, this second island can not be interpreted as related to a secondary reconnecting instability of the kind discussed in Refs. $[18,46,31,21]$ or in the framework of the plasmoid instability scenario $[42,40,41]$, since its appearance is not due to the generation, by the primary tearing mode, of a new current sheet profile on which new modes can be destabilized: the particular choice of the perturbation (40) makes the $m=2$ mode grow even faster than the initially excited $m=1$ perturbation on this equilibrium profile. However, as appears evident from Fig.3, the initial relative amplitude of the $m=1$ and $m=2$ modes is such that the $m=1$ mode saturates and reaches a macroscopic size while the $m=2$ mode still behaves as a linear perturbation with respect to the equilibrium profile, so that the linear growth rate of the latter results strongly perturbed by the nonlinear interaction with the former, already at $t \simeq 23$. Also, the dynamics at the $O$-point of the $m=1$ island is (non evidently, from Figs.4-5) perturbed by the contribution of one of the nascent $m=2$ islands growing in the same position. These complex features, not easy to be discerned when a large spectrum of modes with comparable initial amplitudes are excited, possibly also with different phases, are likely to influence the number of plasmoids observed both in reconnection of large aspect ratio current sheets and in simulations of secondary reconnection to a primary tearing-type mode. 


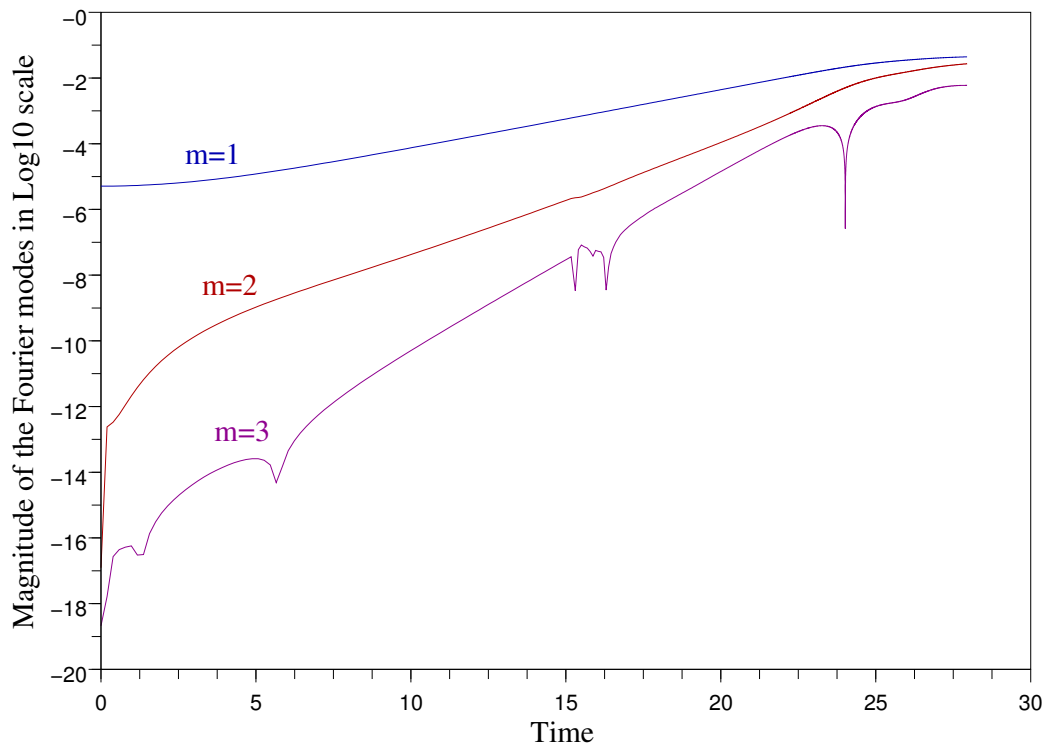

Figure 3: Time evolution for $t \leq 28.2$ of the log-scale amplitude, averaged over the $x$ coordinate, of the first three $k_{y}$-modes corresponding to $m=1,2$ and 3 respectively (equilibrium quantities have log-scale amplitude 0). Simulation parameters: $a=0.5$, $d_{e}=0.3 a, L_{x}=L_{y}=2 \pi$. 


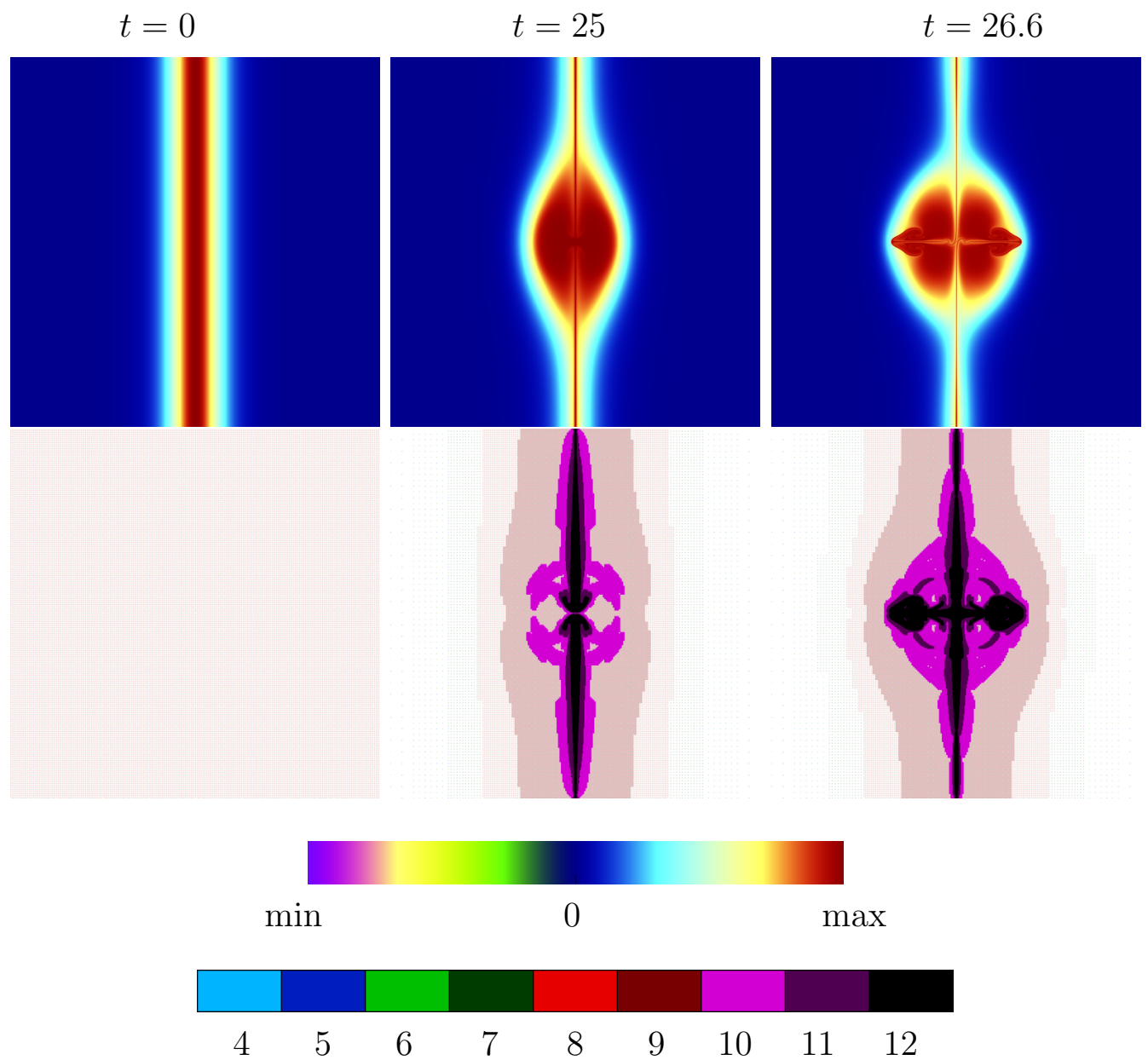

Figure 4: First row: contour plots of the field $F$ at times $t=0, t=25, t=26.6$. Second row: the adaptive grid at the same times, with colors corresponding to the refinement $h=2^{-j} L$ for $4 \leq j \leq 12$. Simulation parameters: $a=0.5, d_{e}=0.3 a, L_{x}=L_{y}=2 \pi$. 


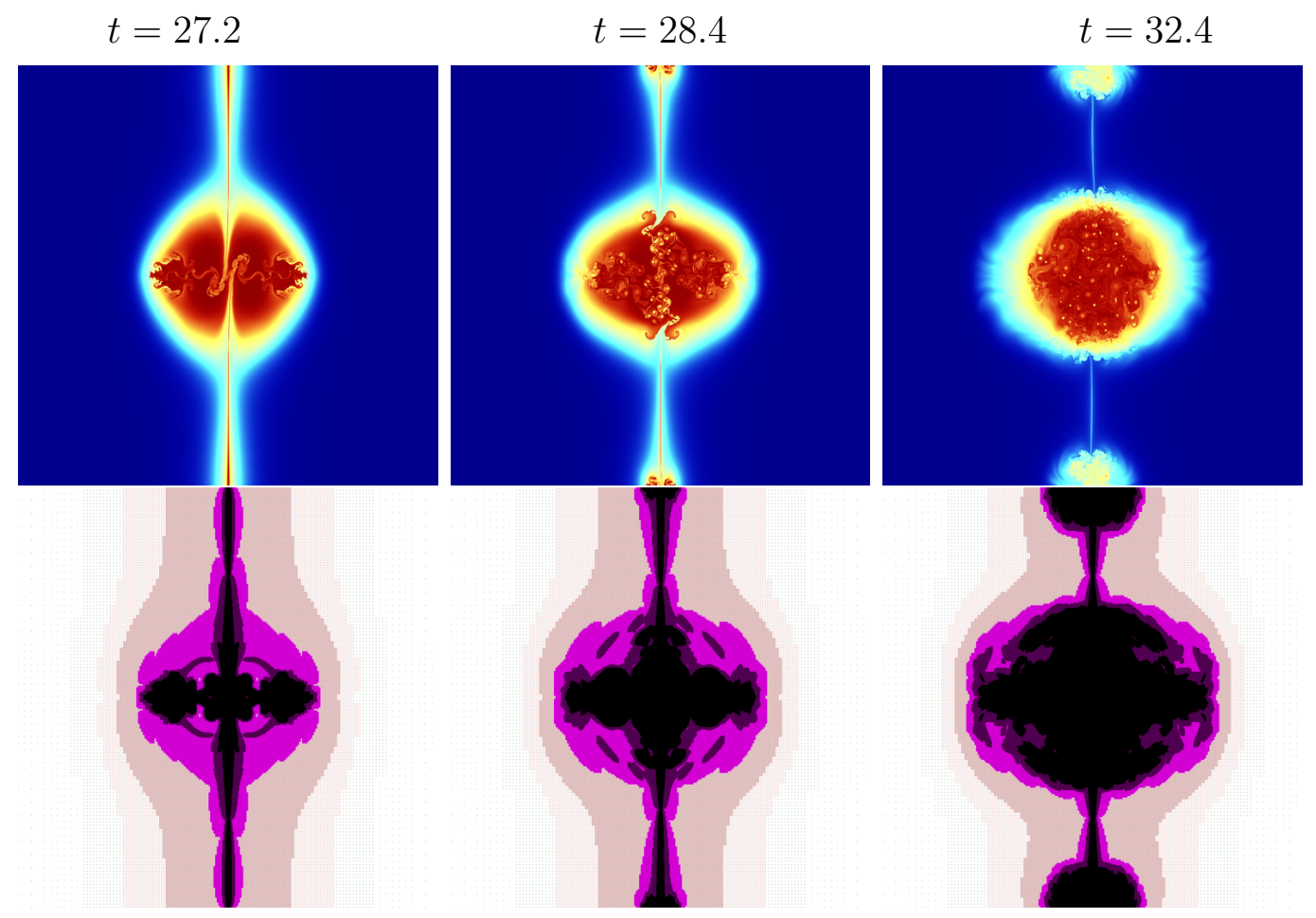

Figure 5: Same as figure 4 but for times $t=27.2, t=28.4, t=32.4$. First row: $F$. Second row: the adaptive grid. 




Figure 6: Total number of points in the adaptive grid as a function of time for the simulation of Figs.3-5.

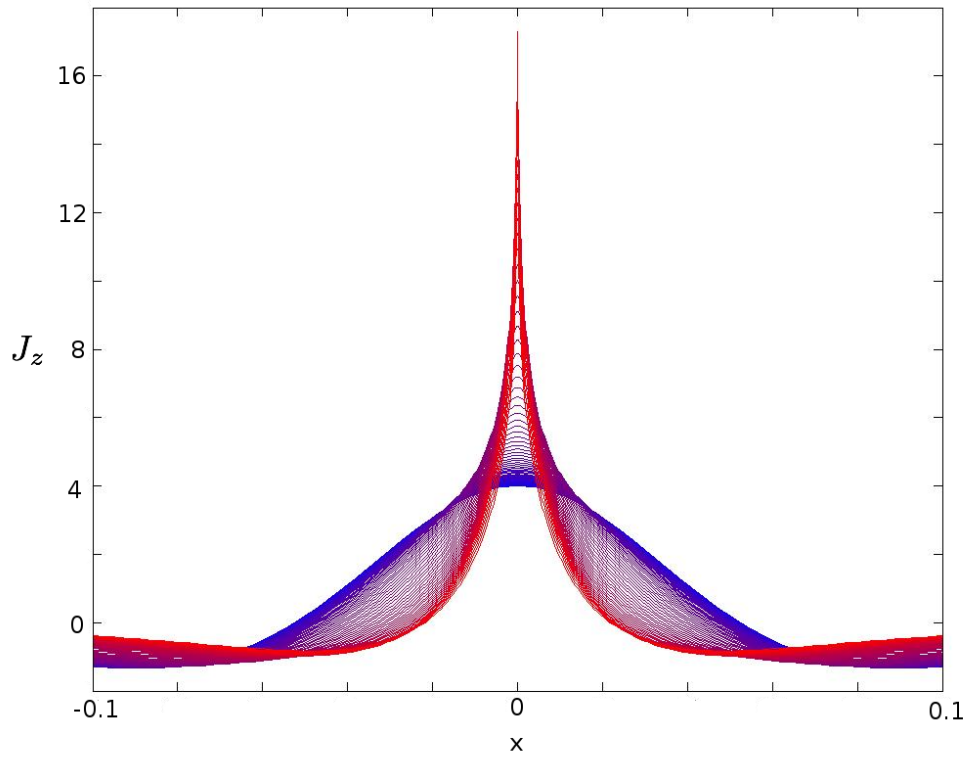

Figure 7: Cut at $y=3 L_{y} / 8$ of the current density $J_{z}$ for the beginning of the experiment $(t \leq 28.2)$ for $-L_{x} / 10 \leq x \leq L_{x} / 10$. The blue color is for $t$ close to 0 and the red one for $t$ close to 28.2 (as time $t$ advances, the $J_{z}$ profile in $x$ peaks at the middle of the domain). Simulation case of Figs.3-5. 


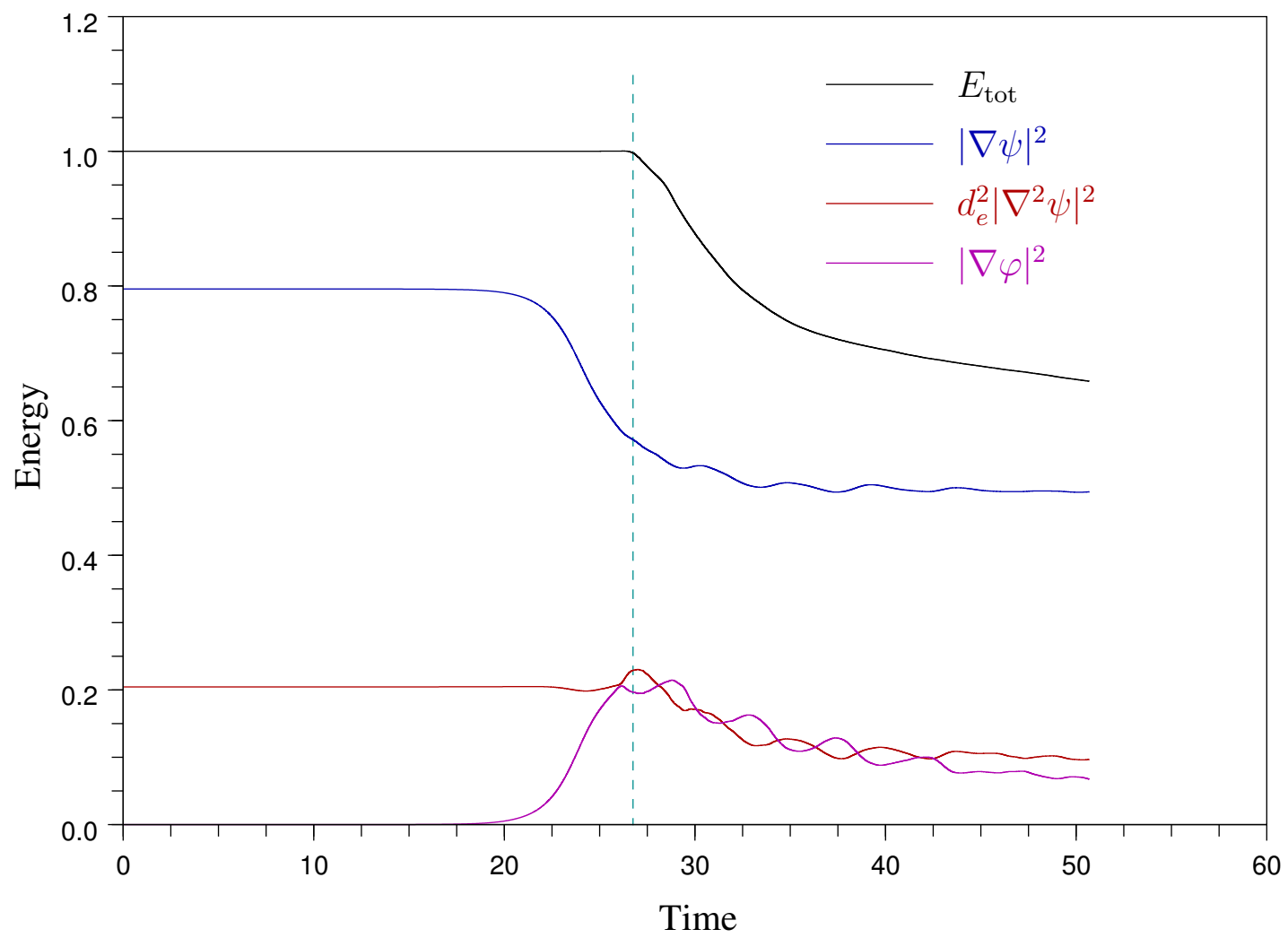

Figure 8: Time evolution of the energy components, normalized to the initial total value of the energy: total energy $E_{\text {tot }}$ of Eq.(8) (black line), magnetic energy $|\nabla \psi|^{2}$ (blue), electron kinetic energy $d_{e}^{2}\left|\nabla^{2} \psi\right|^{2}$ (red), ion kinetic energy $|\nabla \varphi|^{2}$ (mauve). Simulation case of Figs.3-5. 


\subsection{Test case (2): classical tearing with $\rho_{s} \neq 0$}

As a second case we consider reconnection in the Hamiltonian limit for $\rho_{s} \neq 0$. Electron compressibility effects related to $\rho_{s}$ play a stabilizing role $[20,57]$ on the secondary fluid instabilities which are characteristic of the "cold" collision-less regime (Sec.4.1), and the Lagrangian invariants described by Eqs.(20) determine a laminar dynamics of nonlinear reconnection [25], related to the phase-mixing of the scalar fields $F \pm d_{e} \rho_{s} U$ [27].

As a linear benchmark of the code, we have reported in Table 4.2 a few values of the linear growth rate of the mode $k_{y} a=1$, at the varying of $\rho_{s}$, evaluated both directly from simulations with the AMR code and by numerical solution of the eigenvalue problem, following the procedure described at the end of Sec.3.3. These values are further compared with those reported in Ref.[24] and measured in simulations performed with the pseudo-spectral code first used in Ref.[20].

Table 3: Parameters and linear growth rates of the mode $k_{y} a=1$ for different values of $\rho_{s}$ and for $L_{y}=4 \pi a, d_{e}=0.3 a$, with $a=1$. The growth rates $\gamma_{e i g}$ are numerically computed as described at the end of Sec.3.3, whereas the values $\gamma_{n u m}$ in the third column are estimated by tracing the time evolution of the relevant Fourier components $k_{y}$ of the perturbed $\tilde{\psi}$, averaged over the $x$ direction. In the last column, are reported the analogous estimations from output data of simulations run with the pseudo-spectral code used in Ref.[24] (cf. Table 2, there).

\begin{tabular}{|c|c|c||c|}
\hline \hline$\rho_{s}$ & $\gamma_{\text {eig }}$ & $\gamma_{\text {num }}$ & $\gamma_{\text {num }}[24]$ \\
\hline \hline 0.05 & 0.1268 & 0.127 & 0.127 \\
0.1 & 0.1325 & 0.132 & 0.134 \\
0.15 & 0.1408 & 0.139 & 0.140 \\
0.2 & 0.1507 & 0.149 & 0.151 \\
0.3 & 0.1732 & 0.170 & 0.175 \\
\hline
\end{tabular}

As an example of the small scale generation which can be observed in the nonlinear dynamics of this "warm" Hamiltonian regime, we finally present the results of a simulation run for $a=0.5, d_{e}=0.3 a$ and $\rho_{s}=0.6 a$. For this experiment, we take $\alpha=1.3, \epsilon=1.125 e--5$ and $j_{\text {lim }}=11$. Contourplots are shown at different times for the quantity $F$, together with the fields $U$ and $G_{+}$ and with the corresponding levels of refinement of the grid (Fig.9 and 10). The laminar filamentary structures generated by the spatial phase-mixing process, first discussed in Ref.[27], is evidenced in all figures. Analogously to the $\rho_{s}=0$ example considered in case test (1), energy conservation (not 
shown here) is granted as long as the grid refinement keeps up with the spatial filamentation. Again, the loss of spatial resolution also implies a loss of the rotational invariance of the contours. For the choice of a maximal refinement of $h_{l i m}=2^{-11} L$ a loss of spatial resolution appears evident in the three contour-plots at time $t=34.4$.

We finally note that also in this "warm" $\rho_{s}>d_{e}$ case, fluid instabilities of interchange type set in, but this time along the $x$-axis (see the "mushroom" patterns visible in the contourplots of $F$ and $U$ in Fig.10 after time $t=$ 24.). Eventually, then, also KH-type instabilities appear to develop (Fig.10, $t=34.4$ ) on the nonlinearly generated, thin vorticity sheets, analogously to what has been observed for some values of the ion-sound and ion Larmor radius in Ref.[24], although for $\rho_{s}<d_{e}$. The role of flows in the onset of fluid instabilities also in this warm $\rho_{s}>d_{e}$ regime, which apparently has not been pointed out in previous studies, deserves however a more specific, dedicated study, as in the numerical experiment here presented the integral conservations of the Hamiltonian model result too compromised at $t=34.4$. A more specific investigation, probably requiring a better optimized parallel version of the code, would allow to ascertain the interesting possibility to achieve a turbulent regime also in the advanced nonlinear stage of the $\rho_{s}>d_{e}$ case (which, e.g., is more typical of tokamak plasmas). 


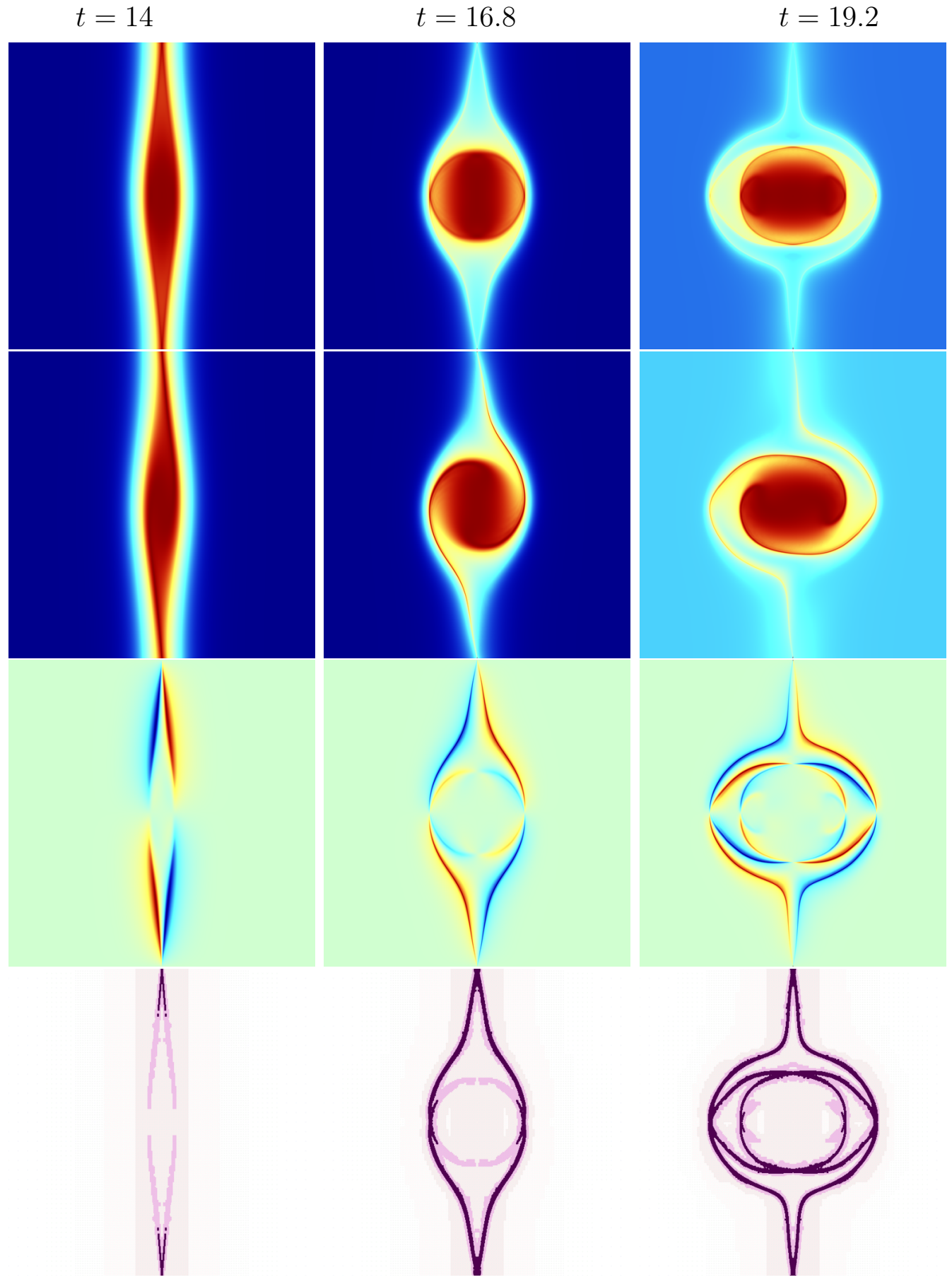

Figure 9: Contourplot of the fields $F$ (first row), $G_{+}$(second row) and $U$ (third row) and of the adaptive grid (bottom row) at times $t=14, t=16.8, t=19.2$. In this simulation the mode $m=1$ has been initially excited in a simulation box with $L_{x}=L_{y}=4 \pi a$, with $d_{e}=0.3 a, \rho_{s}=0.6 a$ and $a=0.5$. 

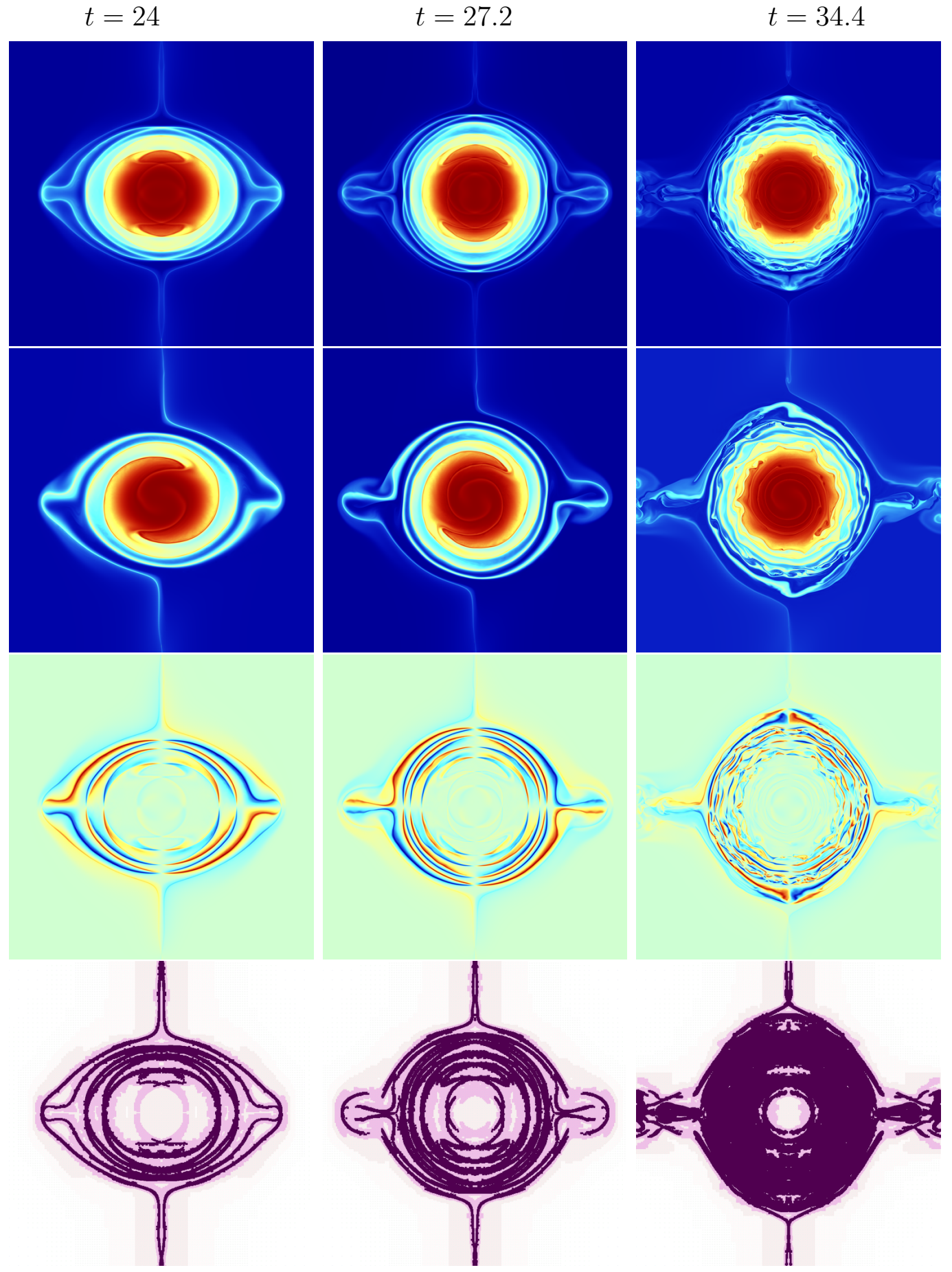

Figure 10: Contourplot of the fields $F$ (first row), $G_{+}$(second row) and $U$ (third row) and of the adaptive grid (bottom row) at times $t=24, t=27.2, t=34.4$. Same simulation run as Fig.9. 


\section{Comments and conclusions}

The study of magnetic reconnection with the reduced magnetohydrodynamic model offers a privileged framework for the development and application of adaptive schemes. These allow in principle to explore parameter ranges otherwise impossible to access with uniform grid schemes.

In this article, we have presented a multigrid adaptive method of high order. We have detailed an original fourth-order multigrid Poisson solver for fully threaded trees. We have accurately addressed the CFL stability of the RMHD equation set, so to allow a remarkable economy of computational resources at the beginning of the numerical experiments and to prevent the onset of numerical instabilities. While the AMR code we have provided is applicable to both the dissipative and strictly collision-less reconnection regimes, we have here focussed on the latter, which is more challenging from the computational point of view, due to the rapid nonlinear generation of small spatial scales, in principle inferiorly unbounded due to the lack of a physical dissipation scale.

We have then presented two numerical experiments performed in this collision-less, Hamiltonian regime, in a parameter range comparable to that of previous numerical results available in literature. Together with a linear validation of the code, performed by measuring some growth rates of a primary tearing mode, we have discussed two examples of nonlinear simulation with which we have addressed two issues of quite general interest in reconnection problems: the role possibly played by the later appearance of primary unstable modes in the interpretation of numerical studies of secondary reconnecting instabilities, and the possibility to achieve a turbulent regime also in "warm" $\left(\rho_{s}>d_{e}\right)$ Hamiltonian reconnection. The numerical experiments here provided are however meant to be just preliminary examples, aimed to highlight the performances and the usefulness of the AMR scheme. The physical issues we have addressed, as well as the applications to the other compelling problems mentioned in Sec.1.1, will be the object of future studies, to be performed with more specific and small $\varepsilon$ physics-oriented simulations.

A MPI parallelized version of this AMR algorithm is in progress. It relies on a hash-table storage of the nodes which are unically designated thanks to a 64-bits marker binded to the node position [88]. This allows the processors to exchange node data without resorting to pointers. 


\section{Appendix A. Algorithm for the Collatz multigrid solver}

We recall that the continuous refinement domains $\bar{\Omega}_{j}$ of level $j$ are embedded: $\bar{\Omega}_{j+1} \subset \bar{\Omega}_{j} \subset \cdots \subset \bar{\Omega}_{0}=\left[-\frac{L}{2}, \frac{L}{2}\right]^{2}$ with periodic boundary conditions. The space step is given by $h_{j}=2^{-j-1} L$, so the discretized domain $\Omega_{j} \subset\left\{\left(-\frac{L}{2}+2^{-j-1} k_{1} L,-\frac{L}{2}+2^{-j-1} k_{2} L\right), 0 \leq k_{1}, k_{2}<2^{j+1}\right\}$ contains $2^{2 j+2}$ points or less.

We also distinguish the interior $\Omega_{j}$ of the domain $\Omega_{j}$ form its boundary $\partial \Omega_{j}: \Omega_{j}=\stackrel{\Omega}{\Omega}_{j} \cup \partial \Omega_{j}$. We denote $\mathbf{x}_{j \kappa} \in \Omega_{j}$ the point at level $j$ and position $-\frac{L}{2}(1,1)+L 2^{-j-1} \boldsymbol{\kappa}$ for $\boldsymbol{\kappa} \in \mathbb{Z} \cap\left[0,2^{j+1}\right)$.

The algorithm of multigrid type uses auxiliary values $r, w$ and $s$ which respectively stand for the residual, the Gauss-Seidel approximant and its cumulation from the different levels. Then the algorithm unfolds as follows:

1. we compute the right member of Eq. (8). For each $j$ and each point $\mathbf{x}_{j \kappa} \in \stackrel{\Omega}{\Omega}_{j}$ we set the perturbed right member

$$
f\left(\mathbf{x}_{j \kappa}\right)=\frac{1}{2}\left[\begin{array}{lll}
0 & 1 & 0 \\
1 & 8 & 1 \\
0 & 1 & 0
\end{array}\right] v\left(\mathbf{x}_{j \kappa}\right) \text {. }
$$

2. We do $n$ iterations of the following loop, with $n \sim 8$ starting from an initial guess issued from the previous time step for instance. This value of $n$ secures the convergence of the iterative process with a final residual much lower than the numerical error.

(a) Restriction: for $j=j_{\max }, \ldots, 1$, with $h=2^{-j-1} L$

i. for all $\mathbf{x}_{j \kappa} \in \AA_{\Omega}$ such that $\mathbf{x}_{j+12 \kappa} \notin \stackrel{\Omega}{\Omega}_{j+1}$, we compute the residual at $\mathbf{x}_{j \kappa}$ :

$$
r\left(\mathbf{x}_{j \kappa}\right)=f\left(\mathbf{x}_{j \kappa}\right)-\frac{1}{h^{2}}\left[\begin{array}{ccc}
1 & 4 & 1 \\
4 & -20 & 4 \\
1 & 4 & 1
\end{array}\right] u\left(\mathbf{x}_{j \kappa}\right),
$$

ii. we initialize an approximate of a solution to Eq. (8) with the residual on the right side

$$
w\left(\mathbf{x}_{j \kappa}\right)=-\frac{h^{2}}{20} r\left(\mathbf{x}_{j \kappa}\right) .
$$


iii. for all $\mathbf{x}_{j \kappa} \in \partial \Omega_{j}$, we set $r\left(\mathbf{x}_{j \kappa}\right)=w\left(\mathbf{x}_{j \kappa}\right)=0$.

iv. we apply $\mu$ ( $=2$ here) Gauss-Seidel iterations on each point $\mathbf{x}_{j \kappa} \in \Omega_{j}$ in a multi-colored order similar to the red-black order of the usual multi-grid Poisson solver i.e. $\left(2 \ell_{1}, 2 \ell_{2}\right)$ then $\left(2 \ell_{1}+1,2 \ell_{2}+1\right)$ then $\left(2 \ell_{1}+1,2 \ell_{2}\right)$ and then $\left(2 \ell_{1}, 2 \ell_{2}+1\right)$ :

$$
w\left(\mathbf{x}_{j \kappa}\right)=w\left(\mathbf{x}_{j \kappa}\right)-\frac{1}{20}\left(h^{2} r\left(\mathbf{x}_{j \kappa}\right)-\left[\begin{array}{ccc}
1 & 4 & 1 \\
4 & -20 & 4 \\
1 & 4 & 1
\end{array}\right] w\left(\mathbf{x}_{j \kappa}\right)\right)
$$

v. we update the residual : for all the points $\mathbf{x}_{j \kappa} \in \Omega_{j}$,

$$
\begin{gathered}
r\left(\mathbf{x}_{j \kappa}\right)=r\left(\mathbf{x}_{j \kappa}\right)-\frac{1}{h^{2}}\left[\begin{array}{ccc}
1 & 4 & 1 \\
4 & -20 & 4 \\
1 & 4 & 1
\end{array}\right] w\left(\mathbf{x}_{j \kappa}\right), \\
u\left(\mathbf{x}_{j \kappa}\right)=u\left(\mathbf{x}_{j \kappa}\right)+w\left(\mathbf{x}_{j \kappa}\right) \text { and } s\left(\mathbf{x}_{j \kappa}\right)=w\left(\mathbf{x}_{j \kappa}\right) .
\end{gathered}
$$

vi. we restrain the residual to pass it to level $j-1$, for all $\mathbf{x}_{j \kappa} \in \Omega_{j}$ such that $\boldsymbol{\kappa}=\left(2 \ell_{1}, 2 \ell_{2}\right)=2 \boldsymbol{\ell}$,

$$
w\left(\mathbf{x}_{j \kappa}\right)=\frac{1}{16}\left[\begin{array}{lll}
1 & 2 & 1 \\
2 & 4 & 2 \\
1 & 2 & 1
\end{array}\right] r\left(\mathbf{x}_{j \kappa}\right)
$$

and then $r\left(\mathbf{x}_{j-1 \ell}\right)=w\left(\mathbf{x}_{j \kappa}\right)$ and $u\left(\mathbf{x}_{j-1 \ell}\right)=u\left(\mathbf{x}_{j \kappa}\right)$.

(b) at level $j=0$, we solve the system exactly

$$
\frac{1}{h^{2}}\left[\begin{array}{ccc}
1 & 4 & 1 \\
4 & -20 & 4 \\
1 & 4 & 1
\end{array}\right] s\left(\mathbf{x}_{0 \kappa}\right)=r\left(\mathbf{x}_{0 \kappa}\right)
$$

we put $u\left(\mathbf{x}_{0 \kappa}\right)=s\left(\mathbf{x}_{0 \kappa}\right)$ and in the periodic case we take away the constant: for all $j$ and $\boldsymbol{\kappa}, f\left(\mathbf{x}_{j \kappa}\right)=f\left(\mathbf{x}_{j \kappa}\right)-\langle r\rangle$.

(c) Prolongation: for $j=1 \ldots j_{\max }$

i. for all $\mathbf{x}_{j \kappa} \in \Omega_{j}$ we add the approximation obtained at level $j-1$ to the solution $u\left(\mathbf{x}_{j \kappa}\right)$ and to the loop approximant $s\left(\mathbf{x}_{j \kappa}\right)$ : 
$w\left(\mathbf{x}_{j 2 \ell}\right)=s\left(\mathbf{x}_{j-1 \ell}\right), w\left(\mathbf{x}_{j 2 \ell+\boldsymbol{e}_{1}}\right)=\frac{1}{2}\left(s\left(\mathbf{x}_{j-1 \ell}\right)+s\left(\mathbf{x}_{j-1 \boldsymbol{\ell}+\boldsymbol{e}_{1}}\right)\right)$ and the same for $\boldsymbol{e}_{2}$ (with $\left(\boldsymbol{e}_{1}, \boldsymbol{e}_{2}\right)$ the canonical basis of $\mathbb{R}^{2}$ ),

$w\left(\mathbf{x}_{j 2 \ell+\boldsymbol{e}_{1}+\boldsymbol{e}_{2}}\right)=\frac{1}{4}\left(s\left(\mathbf{x}_{j-1 \boldsymbol{\ell}}\right)+s\left(\mathbf{x}_{j-1 \boldsymbol{\ell}+\boldsymbol{e}_{1}}\right)+s\left(\mathbf{x}_{j-1 \boldsymbol{\ell}+\boldsymbol{e}_{2}}\right)+s\left(\mathbf{x}_{j-1 \boldsymbol{\ell}+\boldsymbol{e}_{1}+\boldsymbol{e}_{2}}\right)\right)$,

then $u\left(\mathbf{x}_{j \kappa}\right)=u\left(\mathbf{x}_{j \kappa}\right)+w\left(\mathbf{x}_{j \kappa}\right)$ and $s\left(\mathbf{x}_{j \kappa}\right)=s\left(\mathbf{x}_{j \kappa}\right)+w\left(\mathbf{x}_{j \kappa}\right)$.

ii. we interpolate $u$ at the boundary $\partial \Omega_{j}$ of the domain $\Omega_{j}$ with a sixth order centered scheme:

$$
\begin{aligned}
& u\left(\mathbf{x}_{j 2 \ell}\right)=u\left(\mathbf{x}_{j-1 \ell}\right) \text { and } \\
& u\left(\mathbf{x}_{j 2 \ell+\boldsymbol{e}_{1}}\right)=\frac{1}{256}\left[\begin{array}{llllll}
3 & -25 & 150 & 150 & -25 & 3
\end{array}\right] u\left(\mathbf{x}_{j-1 \ell}\right),
\end{aligned}
$$

and the same for $\boldsymbol{e}_{2}$. Knowing that for symmetry reasons we deactivated all the points of the type $\mathbf{x}_{j} 2 \boldsymbol{\ell}+\boldsymbol{e}_{1}+\boldsymbol{e}_{2}$ remaining to the boundary $\partial \Omega_{j}$, we do not need to interpolate them.

3 . in the periodic case at the end of the algorithm we subtract the average of $u: u\left(\mathbf{x}_{j \kappa}\right)=u\left(\mathbf{x}_{j \kappa}\right)-\langle u\rangle$.

\section{Appendix B. Von Neumann stability analysis}

Let us write the von Neumann stability analysis of the RMHD equations Eq. (1), (2) and (3) in the most general case. Then we will set $\eta$ and $\nu$ to zero.

We consider a perturbation (labelled by " ") of the initial solution and consider the new numerical solution: $F+\tilde{F}, U+\tilde{U}, \phi+\tilde{\phi}, \psi+\tilde{\psi}$ and $J_{z}+\tilde{J}_{z}$. In order to explicit the bilinearity of the Poisson brackets, we introduce the linear operator $\nabla^{\perp}$ :

$$
\nabla^{\perp} \varphi \equiv \nabla \times\left(\varphi \boldsymbol{e}_{z}\right)=\boldsymbol{e}_{z} \times \boldsymbol{\nabla} \varphi=\left(-\partial_{y} \varphi, \partial_{x} \varphi\right),
$$

so

$$
[f, g]=\nabla^{\perp} f \cdot \nabla g .
$$

Then, the linearized equations for the perturbed solutions are:

$$
\begin{aligned}
& \partial_{t} \tilde{F}+\nabla^{\perp} \varphi \cdot \nabla \tilde{F}+\nabla^{\perp} \tilde{\varphi} \cdot \nabla F=\rho_{s}^{2}\left(\nabla^{\perp} \tilde{U} \cdot \nabla \psi+\nabla^{\perp} U \cdot \nabla \tilde{\psi}\right)-\eta \tilde{J}_{z}, \\
& \partial_{t} \tilde{U}+\nabla^{\perp} \varphi \cdot \nabla \tilde{U}+\nabla^{\perp} \tilde{\varphi} \cdot \nabla U=-\nabla^{\perp} \psi \cdot \nabla \tilde{J}_{z}-\nabla^{\perp} \tilde{\psi} \cdot \nabla J_{z}+\nu \nabla^{2} \tilde{U}
\end{aligned}
$$


In the framework of von Neumann stability analysis, we assume for a given $\boldsymbol{k}=\left(k_{x}, k_{y}\right)$ that $\tilde{F}(x, y)=\tilde{F}_{k_{x}, k_{y}} e^{i k_{x} x+i k_{y} y}$ and $\tilde{U}(x, y)=\tilde{U}_{k_{x}, k_{y}} e^{i k_{x} x+i k_{y} y}$. The other perturbed functions $\tilde{\varphi}, \tilde{\psi}$ and $\tilde{J}_{z}$ are expressed in terms of $\tilde{F}$ and $\tilde{U}$ by means of Eqs. $(3,6)$ :

$$
\tilde{\varphi}=-\frac{\tilde{U}}{|\boldsymbol{k}|^{2}}, \quad \tilde{\psi}=\frac{\tilde{F}}{1+d_{e}^{2}|\boldsymbol{k}|^{2}}, \quad \tilde{J}_{z}=\frac{|\boldsymbol{k}|^{2}}{1+d_{e}^{2}|\boldsymbol{k}|^{2}} \tilde{F}, \quad \boldsymbol{\nabla} \tilde{f}=i \boldsymbol{k} \tilde{f} .
$$

Using $\nabla^{\perp} f \cdot \nabla g=-\nabla^{\perp} g \cdot \nabla f$, we obtain the linear equation:

$$
\partial_{t}\left(\begin{array}{c}
\tilde{F} \\
\tilde{U}
\end{array}\right)=\boldsymbol{M}\left(\begin{array}{c}
\tilde{F} \\
\tilde{U}
\end{array}\right)
$$

where

$$
\boldsymbol{M} \equiv\left[\begin{array}{cc}
-\boldsymbol{\nabla}^{\perp} \varphi \cdot i \boldsymbol{k}+\frac{\rho_{s}^{2} \boldsymbol{\nabla}^{\perp} U \cdot i \boldsymbol{k}-\eta|\boldsymbol{k}|^{2}}{1+d_{e}^{2}|\boldsymbol{k}|^{2}} & -\frac{\boldsymbol{\nabla}^{\perp} F \cdot i \boldsymbol{k}}{|\boldsymbol{k}|^{2}}-\rho_{s}^{2} \boldsymbol{\nabla}^{\perp} \psi \cdot i \boldsymbol{k} \\
\frac{-|\boldsymbol{k}|^{2} \boldsymbol{\nabla}^{\perp} \psi \cdot i \boldsymbol{k}+\boldsymbol{\nabla}^{\perp} J_{z} \cdot i \boldsymbol{k}}{1+d_{e}^{2}|\boldsymbol{k}|^{2}} & -\boldsymbol{\nabla}^{\perp} \varphi \cdot i \boldsymbol{k}-\frac{\boldsymbol{\nabla}^{\perp} U \cdot i \boldsymbol{k}}{|\boldsymbol{k}|^{2}}-|\boldsymbol{k}|^{2} \nu
\end{array}\right] .
$$

Then we remove the viscous terms (i.e. $\eta=\nu=0$ ). Taking $\left|\left(k_{x}, k_{y}\right)\right| \gg 1$, the matrix $M$ simplifies to:

$$
\boldsymbol{M} \equiv\left[\begin{array}{cc}
-\boldsymbol{\nabla}^{\perp} \varphi \cdot i \boldsymbol{k}+\frac{\rho_{s}^{2} \boldsymbol{\nabla}^{\perp} U \cdot i \boldsymbol{k}}{1+d_{e}^{2}|\boldsymbol{k}|^{2}} & -\frac{\boldsymbol{\nabla}^{\perp} F \cdot i \boldsymbol{k}}{|\boldsymbol{k}|^{2}}-\rho_{s}^{2} \boldsymbol{\nabla}^{\perp} \psi \cdot i \boldsymbol{k} \\
\frac{-|\boldsymbol{k}|^{2} \boldsymbol{\nabla}^{\perp} \psi \cdot i \boldsymbol{k}}{1+d_{e}^{2}|\boldsymbol{k}|^{2}} & -\boldsymbol{\nabla}^{\perp} \varphi \cdot i \boldsymbol{k}
\end{array}\right] .
$$

We assume $\nabla^{\perp} \psi=\nabla^{\perp} F+\varepsilon$ with $|\varepsilon| \ll\left|\nabla^{\perp} F\right|$, which is satisfied at the beginning of the simulation since $F=\left(1-d_{e}^{2} \nabla^{2}\right) \psi$ with $d_{e} \ll 1$, as required by the ideal MHD limit and $\psi$ smooth. This approximation does not impact the stability condition and simplifies a lot the mathematical expressions. Then the trace and the determinant of the matrix $\boldsymbol{M}$ of Eq.(B.8) satisfy

$$
\lambda_{+}+\lambda_{-}=\operatorname{Tr}(\boldsymbol{M})=-2 \boldsymbol{\nabla}^{\perp} \varphi \cdot i \boldsymbol{k}+\frac{\rho_{s}^{2} \boldsymbol{\nabla}^{\perp} U \cdot i \boldsymbol{k}}{1+d_{e}^{2}|\boldsymbol{k}|^{2}},
$$




$$
\begin{aligned}
\lambda_{+} \lambda_{-}=\operatorname{Det}(\boldsymbol{M})= & \left(\boldsymbol{\nabla}^{\perp} \varphi \cdot i \boldsymbol{k}\right)^{2}-\frac{\rho_{s}^{2} \boldsymbol{\nabla}^{\perp} U \cdot i \boldsymbol{k}}{1+d_{e}^{2}|\boldsymbol{k}|^{2}} \nabla^{\perp} \varphi \cdot i \boldsymbol{k}-\frac{\left(\boldsymbol{\nabla}^{\perp} F \cdot i \boldsymbol{k}\right)^{2}}{1+d_{e}^{2}|\boldsymbol{k}|^{2}} \\
& -\rho_{s}^{2} \frac{|\boldsymbol{k}|^{2}}{1+d_{e}^{2}|\boldsymbol{k}|^{2}}\left(\boldsymbol{\nabla}^{\perp} F \cdot i \boldsymbol{k}\right)^{2} .
\end{aligned}
$$

Then, solving

$$
X^{2}-\operatorname{Tr}(\boldsymbol{M}) X+\operatorname{Det}(\boldsymbol{M})=0,
$$

gives the discriminant:

$$
\Delta=\frac{\rho_{s}^{4}\left(\nabla^{\perp} U \cdot i \boldsymbol{k}\right)^{2}}{\left(1+d_{e}^{2}|\boldsymbol{k}|^{2}\right)^{2}}+4 \rho_{s}^{2} \frac{|\boldsymbol{k}|^{2}}{1+d_{e}^{2}|\boldsymbol{k}|^{2}}\left(\nabla^{\perp} F \cdot i \boldsymbol{k}\right)^{2}+4 \frac{\left(\boldsymbol{\nabla}^{\perp} F \cdot i \boldsymbol{k}\right)^{2}}{1+d_{e}^{2}|\boldsymbol{k}|^{2}} .
$$

Hence, the eigenvalues of the matrix $\boldsymbol{M}$ are:

$$
\lambda_{ \pm}=-\nabla^{\perp} \varphi \cdot i \boldsymbol{k}+\frac{\rho_{s}^{2} \boldsymbol{\nabla}^{\perp} U \cdot i \boldsymbol{k}}{2\left(1+d_{e}^{2}|\boldsymbol{k}|^{2}\right)} \pm \frac{\sqrt{\Delta}}{2},
$$

that is, using $\sqrt{a^{2}+b^{2}} \leq|a|+|b|$, they locally satisfy:

$$
\left|\lambda_{ \pm}\right| \leq\left|\nabla^{\perp} \varphi \cdot i \boldsymbol{k}\right|+\frac{\rho_{s}^{2}\left|\nabla^{\perp} U \cdot i \boldsymbol{k}\right|}{1+d_{e}^{2}|\boldsymbol{k}|^{2}}+\left(\frac{1+\rho_{s}^{2}|\boldsymbol{k}|^{2}}{1+d_{e}^{2}|\boldsymbol{k}|^{2}}\right)^{1 / 2}\left|\nabla^{\perp} F \cdot i \boldsymbol{k}\right| .
$$

Hence, the condition $\left|\lambda_{ \pm}\right| \Delta t \leq C$ for $|\boldsymbol{k}|_{\ell^{\infty}} \equiv 1 / \Delta x$, where $C$ solely depends on the type of spatial and temporal discretizations, provides the stability condition on the whole domain, which is implied by:

$$
\Delta t \leq C \frac{\Delta x}{\|\boldsymbol{\nabla} \varphi\|_{\infty}+\frac{\rho_{s}^{2} \Delta x^{2}}{\Delta x^{2}+d_{e}^{2}}\|\nabla U\|_{\infty}+\left(\frac{2 \rho_{s}^{2}+\Delta x^{2}}{\Delta x^{2}+d_{e}^{2}}\right)^{1 / 2}\|\nabla F\|_{\infty}},
$$

where $\|\cdot\|_{\infty}=\left\||\cdot| \ell_{\ell^{1}}\right\|_{L^{\infty}(\Omega)}$, and where we used $|\boldsymbol{k}|^{2} \leq|\boldsymbol{k}|_{\ell^{\infty}}^{2} \leq 2|\boldsymbol{k}|^{2}$, $|\nabla f \cdot i \boldsymbol{k}| \leq|\nabla f|_{\ell^{1}}|\boldsymbol{k}|_{\ell^{\infty}}$ and $\left\|\nabla^{\perp} f\right\|=\|\nabla f\|$.

\section{Acknowledgments}

The authors thank Maurizio Ottaviani for stimulating discussions. Fundings from the Fédération Recherche Fusion par Confinement Magnétique (APP 2016) and access to the HPC machines granted by GENCI (Grand Équipement National de Calcul Intensif) for the project t-2015-057437 are also gratefully acknowledged. 


\section{References}

[1] D. Biskamp, Magnetic Reconnection in Plasmas, Cambridge University Press, UK (2000).

[2] U. Ziegler, A three-dimensional Cartesian adaptive mesh code for compressible magnetohydrodynamics, Comp. Phys. Comm. 116 (1999) 65.

[3] T.I. Gombosi, DeZeeuw, D.L. Groth, et al., Magnetospheric configuration for Parker-spiral IMF conditions: Results of a 3D AMR MHD simulation, Advances in Space Research 26 (2000) 139.

[4] R. Samtaney, P. Colella, T.J. Ligocki, et al., An adaptive mesh semiimplicit conservative unsplit method for resistive MHD, Journ. Phys. Conf. Ser. 16 (2005) 40.

[5] B. Fryxell, K. Olson. P. Ricker, F.X. Timmes, M.Zingale, D.Q. Lamb, P. NacNeice, R. Rosner, J.W. Truran, H. Tufo, FLASH: an adaptive mesh hydrodynamics code for modeling astrophysical thermonuclear flashes, ApJL., 131 (2006) 273.

[6] K. Germaschewski, A. Battacharjee, C.-S. Ng, The magnetic reconnection code: an AMR-based fully implicit simulation suite, Numerical Modeling of Space Plasma Flows, Vol. 359, p.151 (2006).

[7] K. Fujimoto, S. Machida, Electromagnetic full particle code with adaptive mesh refinement technique: application to the current sheet evolution, Journ. Comp. Phys. 214 (2006) 550.

[8] C.S. Ng, D. Rosenberg, K. Germaschewski, et al., A comparison of spectral element and finite difference methods using statistically refined nonconforming grids for the MHD island coalescence instability problem, Astrophys. Journ. Supplement Series 177 (2008) 613.

[9] C. Shen, J.M. Qiu, A. Christlieb, Adaptive mesh refinement based on high order finite difference WENO scheme for multi-scale simulations, Journ. Comp. Phys. 230 (2011) 3780.

[10] M.E. Innocenti, G. Lapenta, S. Markidis, et al., A multi level multi domain method for particle in cell plasma simulations, Journ. Comp. Phys., 238 (2013), 115. 
[11] D.S. Balsara and M. Dumbser, Divergence-free MHD on unstructured meshes using high order finite volume schemes based on multidimensional Riemann solvers, J. Comput. Phys. 299 (2015) 687-715.

[12] E. Deriaz, V. Perrier, Direct numerical simulation of turbulence using divergence-free wavelets, SIAM Multiscale Mod. Sim. 7 (2008) 1101.

[13] O.V. Vasilyev, Solving Multi-Dimensional Evolution Problems with Localized Structures Using Second Generation Wavelets, Int. J. Comp. Fluid Dyn., 17 (2003) 151.

[14] D.S. Balsara, Second-Order-accurate Schemes for Magnetohydrodynamics with Divergence-free Reconstruction, Astrophysical Journal Supplement 151 (2004) 149-184.

[15] D. Rossinelli, M. Bergdorf, B. Hejazialhosseini, et al., Wavelet-based adaptive solvers on multi-core architectures for the simulation of complex systems, Euro-Par 2009 Parallel Processing 721-734, Springer Berlin/Heidelberg, 2009.

[16] D.S. Balsara, Divergence-free reconstruction of magnetic fields and WENO schemes for magnetohydrodynamics, D.S. Balsara, J. Comput. Phys., 228 (2009) 5040-5056.

[17] M. Ottaviani, F. Porcelli, Fast nonlinear magnetic reconnection, Phys. Plasmas 2 (1995) 4104.

[18] D. Del Sarto, F. Pucci, A. Tenerani et al., "Ideal" tearing and the transition to fast reconnection in the weakly collisional MHD and EMHD regimes, J. Geophys. Res.-Space Science 121 (2016) 1857.

[19] M. Ottaviani, F. Porcelli, Nonlinear collisionless magnetic reconnection, Phys. Rev. Lett. 71 (1993) 3802.

[20] D. Del Sarto, F. Califano, F. Pegoraro, Secondary instabilities and vortex formation in collisionless fluid magnetic reconnection, Phys. Rev. Lett. 91 (2003) 235001.

[21] N.F. Loureiro, S.C. Cowley, W.D. Dorland, et al., X-point collapse and saturation in the nonlinear tearing mode reconnection, Phys. Rev. Lett. 95 (2005) 235003. 
[22] D. Grasso, L. Margheriti, F. Porcelli, et al., Magnetic islands and spontaneous generation of zonal flows, Plasma Phys. Controll. Fusion, 48 (2006) L87.

[23] D. Grasso, E. Tassi, F.L. Waelbroeck, Nonlinear gyrofluid simulations of collisionless reconnection, Phys. Plasma 17 (2010) 082312.

[24] D. Del Sarto, C. Marchetto, F. Pegoraro et al., Finite Larmor radius effects in the nonlinear dynamics of collisionless magnetic reconnection, Plasma Phys. Controll. Fusion 53 (2011) 035008.

[25] E. Cafaro, D. Grasso, F. Pegoraro, et al., Invariants and geometric structures in nonlinear Hamiltonian magnetic reconnection, Phys. Rev. Lett. 80, (1998) 4430.

[26] D. Grasso, F. Pegoraro, F. Porcelli, et al., Hamiltonian magnetic reconnection, Plasma Phys. Controll. Fusion, 41 (1999), 1497.

[27] D. Grasso, F. Califano, F. Pegoraro, et al., Phase-mixing and island saturation in Hamiltonian reconnection, Phys. Rev. Lett. 86, (2001) 5051 .

[28] M.E. Mandt, R.E. Denton, J.F. Drake, Transition to whistler mediated magnetic reconnection, Geophys. Res. Lett. 21 (1994) 73.

[29] A. Lazarian, E.T. Vishniac, Reconnection in weakly stochastic field, Astrophys. J., 517 (1999) 700.

[30] F. Pucci, M. Velli, Reconnection of quasi-singular current sheets: the "ideal" tearing mode, Astr. J. Lett. 780 (2014) L19.

[31] D. Del Sarto, M. Ottaviani, Secondary fast reconnecting instability in the sawtooth crash, Phys. Plasmas 012102 (2017) 24.

[32] F. Porcelli, Collisionless $m=1$ tearing mode, Phys. Rev. Lett. 71 (1991) 3802.

[33] H.P. Furth, The "mirror instability" for finite particle gyro-radius, in Plasma Physics and Controlled Nuclear Fusion Research, 1962 (IAEA, Vienna, 1962), Vol. I, p.169. 
[34] B. Coppi, "Inertial" instabilities in plasmas, Phys. Res. Lett. 11 (1964) 226.

[35] D. Biskamp, Magnetic reconnection via current sheets, Phys. Plasmas, 29 (1986) 1520.

[36] W. Park, D.A. Monticello, R.B. White, Reconnection rates of magnetic fields including the effects of viscosity, Phys. Fluids, 27 (1984) 137.

[37] A.Y. Aydemir, Nonlinear studies of $m=1$ modes in high temperature plasmas, Phys. Fluids B, 4 (1992) 3469.

[38] S. Tanuma, T. Yokoyama, T. Kudoh, K. Shibata, Two-dimensional magnetohydrodynamic numerical simulations of magnetic reconnection triggered by a supernova shock in the interstellar medium: generation of X-ray gas in the galaxy, Astrophys. J., 551 (2001) 312.

[39] K. Shibata, S. Tanuma, Plasmoid induced-reconnection and fractal reconnection, Earth Planet Sp 53 (2001) 473.

[40] J.F. Drake, M. Swisdak, K.M. Schoeffler, et al., Formation of secondary islands during magnetic reconnection, Geophys. Res. Lett. 33 (2006) L13105.

[41] W. Daughton, H. Karimabadi, Collisionless magnetic reconnection in large-scale electron-positron plasmas, Phys. Plasmas 14 (2007) 072303.

[42] N.F. Loureiro, A.A. Schekochihin, S.C. Cowley, Instability of current sheets and formation of plasmoid chains, Phys. Plasmas 14 (2007) 100703.

[43] A. Bhattacharjee, Y.M. Huang, H. Yang, B. Rogers, Fast reconnection in high-Lundquist-number plasmas due to the plasmoid instability, Phys. Plasmas, 16 (2009) 112102.

[44] T. Tajima and K. Shibata, Plasma Astrophysics, Addison-Wesley, p.229 (1997).

[45] S. Landi, L. Del Zanna, E. Papini, et al., Resistive magnetohydrodynamic simulations of the ideal tearing mode, Astrophys. J. 805 (2015) 131. 
[46] A. Tenerani, M. Velli, A.F. Rappazzo, et al., Magnetic reconnection: recursive current sheet collapse triggered by "ideal" tearing, Astr. J. Lett. 806 (2015) L132.

[47] Q. Yu, S. Günter, K. Lackner, Formation of plasmoids during sawtooth crashes, Nucl. Fusion 54 (2014) 072005.

[48] A. Tenerani, A.F. Rappazzo, M. Velli, et al., The tearing instability of thin current sheets: the transition to fast reconnection in presence of viscosity, Astrophys. J. 813 (2015) 145.

[49] A. Tenerani, M. Velli, F. Pucci, et al., "Ideally" unstable current sheets and the triggering of fast magnetic reconnection, J. Plasma Phys. 82 (2016) 535820501.

[50] S. Landi, E. Papini, L. Del Zanna, et al., Activation of MHD reconnection on ideal timescales, Plasma Phys. Controll. Fusion 59 (2016), 014052 .

[51] P.A. Cassack, J.F. Drake, The impact of microscopic magnetic reconnection on pre-flare energy storage, Astrophys. J. Lett. 707 (2009) L158.

[52] W.H. Matthaeus, S.L. Lamkin, Rapid magnetic reconnection caused by finite amplitude fluctuations, Phys. Fluids 28 (1985) 303.

[53] M. Velli, F. Pucci, F. Rappazzo et al., Models of coronal heating, turbulence and fast reconnection, Philos. Trans. R. Soc. A 373 (2015) 20140262 .

[54] A. Lazarian, G. Eyink, E. Vishniac, G. Kowal, Turbulent reconnection and its implications, Phyl. Trans. R. Soc. A, 373 (2014) 0144.

[55] M. Muraglia, O. Agullo, S. Benkadda, M. Yagi, X. Garbet, A. Sen, Nonlinear dynamics of magnetic islands imbedded in small-scale turbulence, Phys. Rev. Lett., 107 (2011) 095003.

[56] A. Poyé, O. Agullo, A. Smolyakov et al., Generation of a magnetic island by edge turbulence in tokamak plasmas, Phys. Plasmas 22 (2015) 030704. 
[57] D. Del Sarto, F. Califano, F. Pegoraro, Electron parallel compressibility in the nonlinear development of two-dimensional magnetohydrodynamic reconnection, Mod. Phys. Lett. B 20 (2006) 931.

[58] D. Del Sarto, F. Califano, F. Pegoraro, Current layer cascade in electronmagnetohydrodynamic reconnection and electron compressibility effects, Phys. Plasmas 12 (2005) 012317.

[59] F.L. Waelbroeck, E. Tassi, A compressible Hamiltonian electromagnetic gyrofluid model, Commun. Nonlinear. Sci. Numer. Simul. 17 (2012) 2171.

[60] E. Tassi, P.J. Morrison, F.L. Waelbroeck, et al., Hamiltonian formulation and analysis of a collisionless fluid reconnection model, Plasma Phys. Controll. Fusion 50 (2008) 085014.

[61] R. Fitzpatrick, F. Porcelli, Collisionless magnetic reconnection with arbitrary guide field, Phys. Plasmas 11 (2004) 4713.

[62] E. Tassi, P.J. Morrison. D. Grasso, et al., Hamiltonian four-field model for magnetic reconnection: nonlinear dynamics and extension to three dimensions with externally applied fields, Nucl. Fusion 50 (2010) 034007.

[63] A. Cohen, R. DeVore, G. Kerkyacharian and D. Picard, Maximal spaces with given rate of convergence for thresholding algorithms, Appl. Comput. Harmon. Anal. 11 (2001) 167.

[64] Carpenter, M. H., Kennedy, C. A., Bijl, Hester, Viken, S. A., and Vatsa, Veer N., Fourth-order runge-kutta schemes for fluid mechanics applications, Journal of Scientific Computing 25(1) (2005) 157-194.

[65] S. Popinet, Gerris: a tree-based adaptive solver for the incompressible Euler equations in complex geometries, J. Comput. Phys. 190 (2003) 572 .

[66] D.S. Balsara, Multidimensional Riemann problem with self-similar internal structure. Part I Application to hyperbolic conservation laws on structured meshes, J. Comput. Phys. 277 (2014) 163-200. 
[67] D.S. Balsara and M. Dumbser, Multidimensional Riemann problem with self-similar internal structure. Part II Application to hyperbolic conservation laws on unstructured meshes, J. Comput. Phys. 287 (2015) 269-292.

[68] D.S. Balsara and B. Nkonga, Multidimensional Riemann problem with self-similar internal structure part III a multidimensional analogue of the HLLI Riemann solver for conservative hyperbolic systems, J. Comput. Phys. 346 (2017) 25-48.

[69] C. Burstedde, L.C. Wilcox, O. Ghattas, p4est: Scalable algorithms for parallel adaptive mesh refinement on forests of octrees, SIAM J. Sci. Comput. 33 (2011) 1103.

[70] S. Schaffer, Higher order multi-grid methods, Math. of Comp. 43 (1984) 89.

[71] W. Hackbusch, Multi-grid methods and applications, Springer-Verlag, 1985.

[72] J. Zhang, Multigrid method and fourth-order compact scheme for 2D Poisson equation with unequal mesh-size discretization, J. Comput. Phys. 179 (2002) 170-179.

[73] M. Barad, P. Colella, A fourth-order accurate local refinement method for Poissons equation, J. Comput. Phys. 209 (2005) 1.

[74] Q. Zhang, H. Johansen, P. Colella, A fourth-order accurate finitevolume method with structured adaptive mesh refinement for solving the advection-diffusion equation, SIAM J. Sci. Comput., 34 (2012) B179.

[75] J.G. Charney, R. Fjörtoft, J. von Neumann, Numerical Integration of the Barotropic Vorticity Equation, Tellus, 2 (1950) 237.

[76] H.P. Furth, J. Killeen, M.N. Rosenbluth, Finite-resistive instabilities of a sheet-pinch, Phys. Fluids 16, 459 (1963).

[77] H.P. Furth, P.H. Rutherford, H. Selberg, Tearing mode in the cylindrical tokamak, Phys. Fluids 16 (1973) 1054.

[78] G. Ara, B. Basu, B. Coppi, et al., Magnetic reconnection and $m=1$ oscillations in current carrying plasmas, Ann. Phys. 112 (1978) 443. 
[79] F. Pegoraro, T.J. Schep, Quasilinear energy balance of resistive modes, Phys. Fluids 30 (1987) 3506.

[80] S.V. Bulanov, Magnetic reconnection: from MHD to QED, Plasma Phys. Controll. Fusion 59 (2016) 014029.

[81] B.N. Kuvshinov, F. Pegoraro, T.J. Schep, Hamiltonian formulation of low-frequency, nonlinear plasma dynamics, Phys. Lett. A 196, 296 (1994).

[82] T.J. Schep, F. Pegoraro, B.N. Kuvshinov, Generalized two fluid theory of nonlinear magnetic structures, Phys. Plasmas 1 (1994) 2843.

[83] L. Chacón, A. Stainer, A scalable, fully implicit algorithm for the reduced two-field low- $\beta$ extended MHD model, J. Comp. Phys. 326 (2016) 763.

[84] F. Pegoraro, T.J. Schep, Theory of resistive modes in the ballooning representation, Plasma Phys. Controll. Fusion 28 (1986) 647.

[85] F. Porcelli, D. Borgogno, F. Califano, et al., Plasma Phys. Controll. Fus. 44 (2002) B389.

[86] E.G. Harris, On a plasma sheat separating regions of oppositely directed magnetic field, Il Nuovo Cimento 23 (1962) 115.

[87] F. Pegoraro, D. Borgogno, F. Califano, et al., Developments in the theory of collisionless reconnection in magnetic configurations with a strong guide field, Nonlin. Proc. Geophys., 11 (2004) 567.

[88] K. Brix, S. Melian, S. Müller, M. Bachmann, Adaptive multi-resolution Methods: Practical issues on Data Structures, Implementation and Parallelization, ESAIM Proc. 34 151-183, V. Louvet, M. Massot (eds.), 2011. 